\title{
Observations of Neutral Beam and ICRF Tail Ion Losses Due to Alfvén Modes in TFTR
}

\author{
D. S. Darrow, S. J. Zweben, Z. Chang, C. Z. Cheng, E. D. Fredrickson, \\ E. Mazzucato, R. Nazikian, C. K. Phillips, S. Popovichev*, M. H. Redi, \\ R. B. White, J. R. Wilson, and K.-L. Wong \\ Princeton University, Plasma Physics Laboratory \\ Princeton, NJ 08543 \\ USA \\ *Kurchatov Institute, Russian Research Center \\ Moscow \\ Russia
}

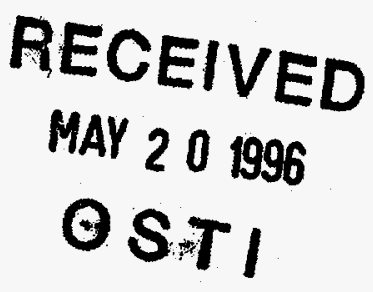

\section{Abstract}

Fast ion losses resulting from MHD modes at the Alfvén frequency, such as the TAE, have been observed in TFTR. The modes have been driven both by neutral beam ions, at low $\mathrm{B}_{\mathrm{T}}$, and by $\mathrm{H}$-minority ICRF tail ions at higher BT. The measurements indicate that the loss rate varies linearly with the mode amplitude, and that the fast ion losses during the mode activity can be significant, e.g. up to $10 \%$ of the input power is lost in the worst case.

\section{Introduction}

In a tokamak, knowledge of the rate of fast ion loss is of importance in determining the energy balance of the discharge. Heating of the discharge may be diminished if losses are significant, since neutral beam ions, ICRF heating tail ions, and alpha particles all heat the plasma and may all be lost through processes which expel fast ions. In addition, a loss of fast ions which is sufficiently intense and localized may cause damage to plasma facing components in the vacuum vessel.[1] For these reasons, knowledge of the fast ion loss mechanisms is desirable.

Loss processes for fast ions in a tokamak fit into two broad categories: single particle and collective. Single particle losses are those, such as first orbit loss, which are independent of the number of fast ions present. These have been seen in numerous instances with various fast ions in TFTR and other tokamaks, and are reported elsewhere [2-7]. Collective losses arise when the fast ion density is sufficient to drive instabilities which then cause loss. The drive can come from $\partial \mathrm{f}_{\mathrm{f}} \partial \psi$ where $\mathrm{f}_{\mathrm{f}}$ is the fast ion distribution function) and $\partial \mathrm{f}_{\mathrm{f}} \partial \mathrm{E}$. Here $\mathrm{E}$ is the particle energy and $\psi$ is the radial (poloidal flux) coordinate. Examples of collective instabilities include the toroidal Alfvén eigenmode (TAE), the kinetic ballooning mode (KBM), alpha driven sawteeth, alpha driven fishbones, Alfvén waves, and ion cyciotron waves. This paper limits itself to the presentation of observations made during Alfvén frequency instabilities in TFTR which were excited under two conditions: at low field (1.5 T), with neutral beam ions driving the mode $[8,9]$, and at intermediate field $(3.4 \mathrm{~T})$, with the hydrogen minority ICRF tail ions driving the mode.[10]

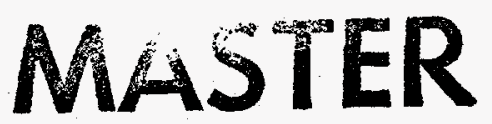


The detectors used to measure the fast ion loss were the escaping alpha detectors, which are described elsewhere $[2-5,11,12]$. These detectors act as magnetic spectrometers, dispersing fast ions onto a scintillator, depending upon their gyroradius and pitch angle. The detectors are located at $20^{\circ}, 45^{\circ}, 60^{\circ}$, and $90^{\circ}$ below the outboard midplane. For the NBI driven mode, only the $20^{\circ}$ probe could be utilized, since the $100 \mathrm{keV}$ deuterons are screened from the other probes by a $3 \mu \mathrm{m}$ thick aluminum foil over the entrance slit which stops particles with energies below $400 \mathrm{keV}$; the $20^{\circ}$ has no such foil. For the ICRF tail ion driven TAE, the $20^{\circ}, 45^{\circ}$, and $60^{\circ}$ probes all showed measurable signals.

The TAE is a global mode in a tokamak. It is characterized by a single toroidal mode number, $n(n \neq 0)$, and several poloidal mode numbers, $\mathrm{m}$, which vary with minor radius. Its frequency is given approximately by $\omega=v_{A} / 2 q R$, where $v_{A}$ is the Alfven velocity. The mode is driven by the spatial gradient of the fast ion density, and in order for the mode to be unstable, the fast ion velocity must exceed $v_{A}$ (although there is some sideband drive of the mode if $v_{f}>v_{A} / 3$ ). In addition, the total drive must exceed the total damping. The experimental observations of the mode seen during ICRF heating match all the characteristics expected of a TAE, hence that name is used here to refer to that mode. The mode observed during neutral beam injection matches most characteristics of the TAE, except that it appears to have a toroidal mode number of $n=0$. Hence, it is referred to here as an "Alfvén mode."

Experiments on TAEs, motivated by the issues mentioned in the first paragraph, were first done with neutral beam plasmas in DIII-D [13-15] and TFTR. $[8,9,16]$ Subsequent results have also come from PBX-M[17], JT-60U[18-20], and JET.[21] The results from JT-60U and JET concentrate predominantly on ICRF minority tail driven TAEs, although the JET work includes some on neutral beam driven modes. Recent work on JET has included external excitation of the TAE by an antenna in order to measure directly the damping of the mode.[22] The results from DIII-D indicated that neutral beam ions were destabilizing TAEs with $2 \leq n \leq 10$, causing up to $70 \%$ loss of the beam ions which, in turn, caused the beam ion beta to saturate. Losses were proportional to the TAE amplitude, and were largest near the midplane. Neutral beam ion driven TAEs were observed in TFTR at low $B_{T}$, with $2 \leq n \leq 4$. A loss of up to $50 \%$ loss of beam ions was inferred from the change in the neutron rate. In PBX-M, modes in the Alfvén frequency range were seen, localized near the $q=1$ surface, and having a ballooning character. The loss of beam ions due to the mode was inferred from a drop in the neutron rate during the mode. JT-60U reported TAEs driven by H-minority ICRF tail ions. The modes occurred with a threshold in power of $\sim 3 \mathrm{MW}$, were localized near the $q=1$ surface, and had $4 \leq \mathrm{n} \leq 12$. The stored energy in the tail ion population was observed to drop by $20 \%$ during the TAEs. In JET, TAEs with $\mathrm{n} \leq 6$ were observed during combined neutral beam injection and ICRF heating. The TAE appeared above $\sim 5 \mathrm{MW}$ of ICRF power, and resulted in a loss of beam ions which was linear with the mode amplitude.

Characteristics of the neutral beam driven Alfvén mode in TFTR and losses produced by it are presented in Section II of this paper. Section III discusses some implications of these results. Section IV describes characteristics of losses from a TAE driven by H-minority ICRF tail ions, while Section V draws implications from those results and Section VI summarizes the paper.

\section{Neutral Beam Ion Driven Alfvén Mode}

The NBI driven Alfvén mode in TFTR appears only when the toroidal field is lowered sufficiently so that the injected $100 \mathrm{keV}$ beam deuterons satisfy $v_{D}^{\sim} v_{A}$. Under these conditions, an instability is 


\section{DISCLAIMER}

Portions of this document may be illegible in electronic image products. Images are produced from the best available original document. 

observed on the Mirnov coils which has a frequency of approximately $120 \mathrm{kHz}$. The mode amplitude increases with increasing NBI power, and the mode vanishes entirely when $B_{T}$ is raised $[8,9]$. Likewise, the frequency scales with density and toroidal field according to the formula for the frequency stated above. Typical parameters for these plasmas are: $1 \mathrm{~T} \leq \mathrm{BT} \leq 2 \mathrm{~T}, 300 \mathrm{kA} \leq \mathrm{I}_{\mathrm{p}} \leq 700 \mathrm{kA}, \mathrm{n}_{\mathrm{e}}=3 \times 10^{19}$ $\mathrm{m}^{-3}, \mathrm{R}=2.40 \mathrm{~m}, \mathrm{a}=0.75 \mathrm{~m}, \beta_{\text {fast }} \sim 0.5 \%$, and $\langle\beta\rangle \sim 1 \%$. For these shots, the aperture of the $20^{\circ}$ probe was located between $R=3.41 \mathrm{~m}(0.32 \mathrm{~m}$ from the plasma edge $)$ and $R=3.51 \mathrm{~m}(0.42 \mathrm{~m}$ from the plasma edge), with it most often being placed at $R=3.46 \mathrm{~m}(0.37 \mathrm{~m}$ from the plasma edge). The limiter radius at the angular location of the probe is $\mathrm{R}=3.525 \mathrm{~m}$.

Figure 1 shows the time histories of a Mirnov coil signal, the neutron rate, and the rate of neutral beam ion loss to the $20^{\circ}$ escaping alpha detector.[16] The bursts in the Mirnov signal are caused by the Alfvén mode. During each burst, the beam ion loss rate increases. The neutron rate is seen to drop after the larger bursts, indicating an expulsion of beam ions. The time delay between the peak of the Mirnov signal and the peak of the beam ion loss is about $0.5 \mathrm{msec}$. This interval corresponds to several tens of bounce times for the trapped beam ions, and indicates that the loss mechanism requires numerous transits of the particle through the mode in order to cause expulsion. In this discharge, the bursts occur at a regular interval and result in approximately equal losses. There is a baseline loss rate to which the signal returns in between the bursts. This baseline loss is of trapped beam ions (see below) and probably results from the few percent of beam neutrals which ionize at a sufficiently large minor radius to be born onto trapped orbits.

Figure 2 compares the frequency spectrum of the Mirnov signal during a burst to the spectrum between bursts. Though the spectrum during a burst is rather broad, it is centered around $120 \mathrm{kHz}$, which is consistent with the expected Alfvén frequency for this plasma. During the Alfvén mode burst, there is also enhanced MHD activity at $25 \mathrm{kHz}$, and some at $\sim 6 \mathrm{kHz}$. The $6 \mathrm{kHz}$ mode is observed in some shots to persist between the bursts, and therefore seems unlikely to be the cause of the bursting losses seen. This low frequency mode exists principally near the center of the plasma, and appears to have mode numbers $n=1$ and $m=1$. Both the $25 \mathrm{kHz}$ and $120 \mathrm{kHz}$ modes have $\mathrm{n}=0$ according to the Mirnov coil array. The Alfvén mode has a poloidal mode number of $m=1$. By way of comparison, TAEs with $4 \leq n$ $\leq 8$ have been observed in TFTR under conditions very similar to these, as described in Refs. 8 and 9 . In DIII-D, neutral beam driven TAEs were frequently accompanied by simultaneous fishbone modes at a lower frequency.[13]

Figure 3 depicts the characteristics of a group of bursts taken from 12 nominally identical shots. In Figure 3a, the amplitude of the drop in neutron rate at each burst, defined in the same way as in Ref. 13, is plotted against the time-integral of the Mirnov amplitude. The integrated Mirnov amplitude is computed by high pass filtering the digitized Mirnov data with a cutoff of $62 \mathrm{kHz}$, then computing its rms amplitude. Following that, the background level of turbulent magnetic fluctuations is subtracted, and the remainder is integrated over the duration of the burst.

Several possible relationships between the loss and the mode amplitude have been predicted by theories, depending upon the particular conditions of the instability and the fast ion velocity space. The predictions are that the loss will vary as $\delta \mathrm{B}^{0.5}, \delta \mathrm{B}$, or $\delta \mathrm{B}^{2}$.[23] Taking the drop in the global neutron rate as a measure of the loss of beam ions, functions of all three forms were least-squares fitted to the data shown in Fig. 3a. The results were correlation coefficients of $0.637,0.692$, and 0.163 respectively. A linear relationship between the drop in the neutron rate and the time integral of the mode amplitude is therefore most consistent with the data. A similar result is obtained in Sec. IV below for ICRF tail ion 
Figure $3 \mathrm{~b}$ shows the time-integrated loss per burst to the midplane probe, again plotted against the time-integrated Mirnov amplitude. Least squares fitting of the functional forms noted above was also applied to this data, resulting in correlation coefficients of $0.513,0.648$, and 0.702 respectively for variation as the square root, first power, and square of the mode amplitude. If one instead assumes that the data should be represented by a linear form with an offset, the correlation coefficient is 0.709 . While the drop in the neutron rate scaled linearly with mode amplitude, the actual losses to the $20^{\circ}$ probe are best represented as varying with the square of the mode amplitude or as varying linearly with mode amplitude above some threshold.

While it is possible that the losses result from the $25 \mathrm{kHz}$ mode, and not from the Alfvén mode, the existing data contradicts that interpretation. Figure $3 \mathrm{c}$ displays the drop in the neutron rate, plotted versus the integrated amplitude of magnetic fluctuations with $10 \mathrm{kHz} \leq \mathrm{f} \leq 62.5 \mathrm{kHz}$. The distribution is more scattered than that shown in Fig. 3a, and the correlation coefficients are significantly lower than those obtained when the $120 \mathrm{kHz}$ mode amplitude is used. Hence, the Alfvén mode seems to have the dominant role in driving the losses.

The pitch angle distribution during the bursts does not change significantly from the distribution between bursts. Figure 4a compares the pitch angle distributions of the losses during and between the bursts. In this and all the figures in this paper, the pitch angles quoted are with respect to the toroidal field. Hence, the pitch angle, $\chi$, is defined as $\chi=\arccos \left(v_{T} / v\right)$. In this figure, the amplitude of the distribution during the Alfvén mode burst is normalized to the amplitude of the distribution between the bursts to facilitate comparison. The pitch angle of the loss both during and between the bursts is similar. In this particular case, the loss shifts to higher pitch angles i.e. more deeply trapped particles during the burst, but this is not uniformly the case. During some bursts in the same shot, the loss is at lower pitch angles. In this case, the loss peaks at a pitch angle of $64^{\circ}$, well into the trapped region of phase space. The pitch angle of the passing/trapped boundary is $47^{\circ}$ at the $20^{\circ}$ probe for these plasma conditions and particle energies. The typical shape of these orbits is depicted in Fig. 5.

The gyroradius distribution during the bursts does not change significantly from the distribution between bursts. Figure $4 \mathrm{~b}$ displays the gyroradius distributions (again normalized to the same amplitude) of the losses during and between bursts. For the burst chosen, the same one as in Fig. 4a, the particles lost during the burst are at slightly lower gyroradii . During other bursts, however, the gyroradius distribution does not change at all from that between the bursts. The gyroradius seen is that expected for birth-energy (100 keV) deuterons.

The orbits of the partieles lost lie in the stochastic ripple loss region. This is shown in Fig. 5, which depicts several orbits computed from the observed pitch angles and energies at the detector. These are for a $420 \mathrm{kA}, 1.4 \mathrm{~T}$ plasma. Also shown in the figure is the stochastic toroidal field ripple diffusion domain for $95 \mathrm{keV}$ deuterons.[24] Any beam ion with banana tips in this domain is subject to stochastic ripple diffusion, which will eventually result in its loss to the wall near the midplane. As shown in this figure, the orbits of the lost particles detected at several probe locations are all subject to stochastic ripple diffusion. Since essentially the same pitch angles are observed in the absence of the mode, the pitch angle data and this plot indicate that the losses in the absence of the mode can be attributed to stochastic ripple diffusion. The fact that additional loss driven by the mode appears at the same pitch angle suggests that the mode-driven loss may result from a two-step process in which the mode moves 
beam ions outward in minor radius until the particles are in the stochastic ripple domain. Then, the ripple diffusion carries these particles out of the plasma. Since the ripple diffusion can take many bounces before the particle is ejected, this conjecture would be consistent with the observed time delay between the peak of the mode amplitude and the peak of the loss. Likewise, it would explain the fact, depicted in Fig. 1, that the losses persist for some time after the mode has vanished. It is also possible that stochastic diffusion can occur as the beam ions encounter an effective ripple in the field due to the magnetic fields of the mode itself (rather than due to the discreteness of the toroidal field coils). Such a process would not explain, however, the persistence of the loss after the mode has vanished.

Figure 6 shows the total loss to the probe versus position. The loss increases as the probe moves farther inward away from the limiter, which is at $R=3.525 \mathrm{~m}$. If the decay of the signal with increasing distance from the plasma is fitted to an exponential function, the result is an e-folding distance of $\sim 6 \mathrm{~cm}$.

Figure 7 depicts the peak pitch angle and the observed energy of the lost deuterons as a function of probe position. The peak pitch angle varies by $\sim 5.5^{\circ}$ over the $7 \mathrm{~cm}$ which the probe is scanned. The apparent energy varies from about $65 \mathrm{keV}$ to $100 \mathrm{keV}$, depending upon position. The observed decrease in energy as the probe moves away from the plasma may be due to the TAE extracting more energy from the beam ions as they move outward. Collisional slowing down proceeds too slowly to account for this reduction of energy, since the losses at all positions show the same time scales as are depicted in Fig. 1.

\section{Discussion of NBI Loss Results}

The regular bursting behavior depicted in Fig. 1 is consistent with a cyclical process of increasing the population of fast ions up to a point above the instability threshold, whereupon the instability sets in, ejects the fast ions, and falls below threshold.[25]

Due to their relatively low density, the neutron production in the discharges considered here is predominantly ( $80 \%$ according to the SNAP transport code) from reactions of beam ions with other beam ions, i.e. $S_{n} \propto n_{f}{ }^{2}$. In contrast, the neutron production in the DIII-D experiments is computed to be dominantly from beam-plasma reactions.[13] Each individual burst of the TAE produces a reduction in the neutron rate of between $5 \%$ and $10 \%$, so the change in beam ion density can be treated perturbatively: $\mathrm{S}_{\mathrm{n}} \propto \mathrm{n}_{\mathrm{f}}{ }^{2}=\mathrm{n}_{\mathrm{f}}{ }^{2}\left(1-\delta \mathrm{n}_{\mathrm{f}} / \mathrm{n}_{\mathrm{f} 0}\right)^{2}$. (This zero-dimensional model ignores the radial dependence of the fast ion density.) The result is that the change in the neutron rate, to lowest order, is linear in the change in the beam ion density: $\delta S_{n} \propto-2 n_{f o} \delta n_{f}$. This means, then, that the change in neutron rate, the vertical axis in Fig. 3a, can be regarded as directly proportional to the change in the beam ion density, i.e. the amount of loss. Thus, the fact that a straight line best fits the data in Fig. 3a implies that the fast ion loss is linearly proportional to the TAE amplitude.

Although the neutron data implies that the fast ion loss is linearly proportional to the mode amplitude, the direct measurements of loss from the $20^{\circ}$ probe seem best fit by the square of the mode amplitude. This discrepancy may be resolved if one assumes that there is a threshold in mode amplitude below which particles are not transported to the edge of the plasma. This assumption can be made plausible by recalling that the neutron rate can be diminished simply by moving beam ions farther outward in minor radius, where they are less reactive, whereas particles must be moved all the way to the edge before they can be seen by the $20^{\circ}$ probe. If the losses are attributable to stochastic ripple diffusion, this onset threshold may represent the point at which beam ions are transported into the stochastic ripple domain. 
If one accepts this view, then a consistent interpretation of both loss measurements is that the magnitude of the beam ion loss scales linearly with mode amplitude. A similar linear dependence of fast ion loss on mode amplitude has been observed in JET as well.[21]

It is possible to cross-calibrate the $20^{\circ}$ detector from the $90^{\circ}$ detector under other quiescent discharge conditions where fusion product loss dominates.[5] Assuming the Scintillator response is linear with the fast ion power incident upon it, that calibration can be used for neutral beam losses as well. For a typical discharge with a bursting frequency as shown in Fig. 1, the TAE bursts result in losses to the wall of $\sim 0.06 \mathrm{~W} / \mathrm{cm}^{2}$. Knowing that these losses result from stochastic toroidal field ripple diffusion, the total loss can be crudely estimated by assuming this loss rate holds between $0^{\circ}$ and $30^{\circ}$ below the outboard midplane, and is axisymmetric. The result is that total losses are estimated to be $\sim 6 \mathrm{~kW}$, or $-0.06 \%$ of the injected power of $10 \mathrm{MW}$. This result is not consistent with the change in the neutron rate produced by the bursts, which indicate $\sim 10 \%$ beam ion loss. There are several assumptions used in these estimates, that may be erroneous. First, the loss rate inferred from the change in the neutron rate assumes that the particles have actually been lost from the plasma, when they may only have been displaced to some region where they produce fewer neutrons. Second, the loss rate inferred from the probe is based on an assumption about the poloidal distribution of the loss, which may be quite different from the actual distribution. Finally, the probe used cannot detect particles with pitch angles below $40^{\circ}$, which may lead to an underestimate of the losses if particles in this pitch angle range are lost in quantity. In fact, most of the beam ions in the plasma do lie below this cutoff in pitch angle. Measurements in DIII-D indicate much higher beam ion loss powers than this power inferred from the probe measurement.[13]

\section{H-minority ICRF Tail Driven TAE}

Another condition in TFTR in which TAEs can be driven is during H-minority ICRF heating $[10,26]$. In such plasmas, the tail ions can exceed the Alfvén velocity and drive TAEs. Typical plasma parameters are: $\mathrm{B}_{\mathrm{T}}=3.4 \mathrm{~T}, 1.3 \mathrm{MA} \leq \mathrm{I}_{\mathrm{p}} \leq 1.8 \mathrm{MA}, \mathrm{R}=2.62 \mathrm{~m}, \mathrm{a}=0.98 \mathrm{~m},{ }^{4} \mathrm{He}$ or $\mathrm{D}$ plasmas, $\left\langle\mathrm{n}_{\mathrm{e}}\right\rangle=2.5 \times 10^{19}$ $\mathrm{m}^{-3}, 3 \mathrm{MW} \leq \mathrm{PICRF} \leq 11 \mathrm{MW}, \mathrm{T}_{\text {tail }}=300-600 \mathrm{keV}\left(\mathrm{v}_{\text {tail }} \sim \mathrm{v}_{\mathrm{A}} \sim 1 \times 10^{7} \mathrm{~m} / \mathrm{sec}\right),\langle\beta\rangle=0.3 \%$, and $\left\langle\beta_{\text {fast }}\right\rangle$ $=0.03 \%$.

Under these conditions, the TAE appears as a magnetic fluctuation at a frequency between 170 and 250 $\mathrm{kHz}$, with $4 \leq \mathrm{n} \leq 10$. Figure 8 shows a typical frequency spectrum for such a mode, which often includes several modes very closely spaced in frequency. The mode is also seen with the microwave reflectometer [10] and it appears only when the ICRF power exceeds $3 \mathrm{MW}$ (equivalently, when the fast ion tail stored energy exceeds $70 \mathrm{~kJ}$ ). The mode frequency scales inversely with the square root of plasma density, in agreement with the equation for the Alfvén frequency. Its scaling with magnetic field cannot be checked convincingly since changing $B_{T}$ also alters the ICRF resonance location. Similar behaviors of mode onset and frequency scaling have also been observed during minority ICRF heating in JET and JT-60U.[18-21]

H-minority tail ion losses result from the TAE. These are most easily measured by the $45^{\circ}$ and $60^{\circ}$ probes which are in fixed locations, have apertures closer to the plasma than the $20^{\circ}$ probe, and are better protected from the plasma heat flux than is the $20^{\circ}$ probe. Figure 9 displays the time history of a TAE driven by the H-minority tail ions. The top trace depicts the total fast ion loss to the $45^{\circ}$ escaping alpha prcbe versus time. The second trace is the mode amplitude. When the mode appears, the fast ion loss is seen to increase suddenly and substantially. As time progresses, the mode amplitude diminishes, 
as do the fast ion losses. This, presumably, is due to some evolution of the tail ion distribution. A similar behavior is seen in JET.[21] At the sawtooth crashes (seen in the third trace) the mode momentarily turns on again, and the losses increase correspondingly. This, it is postulated, is due to a rearrangement of the fast ion profile. This figure also shows the turn-on threshold of $3 \mathrm{MW}$. The tail ions which are lost are trapped ions whose energies lie in the range 0.5 to $1 \mathrm{MeV}$, based upon the gyroradii detected. The lower end of this range, $0.5 \mathrm{MeV}$, corresponds to protons whose velocities are approximately equal to the Alfvén velocity, as noted above.

Figure 10(a) depicts the mode amplitude, as determined from the Mirnov coils, and the escaping tail ion loss power as functions of the applied ICRF power. Both quantities increase approximately linearly with the applied ICRF power (more easily seen in Fig. 10(b)). The escaping tail ion loss power is estimated assuming that the poloidal distribution of the losses is the same as that of first orbit loss. This assumption is known to be incorrect, since tail ion losses at the $90^{\circ}$ detector position are negligible, while first orbit loss there is not. However, it does provide the best existing absolute calibration of the $45^{\circ}$ detector. Comparison of the fast ion loss signal in the $45^{\circ}$ detector during the TAEs to the DD fusion product loss signal during an ordinary neutral beam heated shot yields an absolute magnitude for the losses produced by the TAE. Because of the number of assumptions in this process, the uncertainties in the power loss quoted in Fig. 10(a) are accurate only to within an order of magnitude. In spite of this uncertainty, it is apparent that a significant amount of power is lost in ICRF tail ions when the TAE amplitude is large. In the worst case shown, with $11 \mathrm{MW}$ of applied ICRF power, an estimated 1 MW is lost due to escaping tail ions.

This substantial loss may help explain two other experimental observations. First, it is seen that the energy stored in fast ions does not increase with ICRF power for powers above $5 \mathrm{MW}$ in ${ }^{4} \mathrm{He}$ majority plasmas.[26] It may be that part or all of this additional input power is rapidly lost to the walls in tail ions (the accuracy of the measurements described above does not preclude the possibility that all the additional input power above $5 \mathrm{MW}$ is lost in escaping tail ions). Secondly, some in-vessel components in TFTR which lie $\sim 10 \mathrm{~cm}$ in the shadow of the limiters have been melted or damaged due to excessive heating. This damage is concentrated near the midplane and corresponds to where most of the tail ions should be lost due to the TAE, as described below.

Figure 10(b) depicts the same data as in 10(a), but plotted as power loss versus mode amplitude. The relationship between the two quantities is best fit by a straight line. This is consistent with the relationship between mode amplitude and losses seen in the NBI-driven case described above. This linearity is again in agreement with reported JET results.[21]

Based upon the data from the $90^{\circ}, 60^{\circ}$, and $45^{\circ}$ detectors, the loss of tail ions increases toward the midplane. Unfortunately, the $20^{\circ}$ detector is of a different geometry than the others, with its aperture 3 $\mathrm{cm}$ back from the probe tip, rather than $1 \mathrm{~cm}$ for the others. This difference makes it difficult to compare the fluxes in each probe directly. The loss versus poloidal position of the probes is plotted in Fig. 11 , and shows this trend of losses increasing rapidly toward the midplane. This poloidal distribution is consistent with a loss process which causes a finite-sized radial step within one bounce time.

Figure 12 shows the orbits of particles which enter the $20^{\circ}, 45^{\circ}$, and $60^{\circ}$ detectors with and without the TAE (the $90^{\circ}$ detector sees a negligible signal). The ICRF resonance layer is shown as a vertical dashed line in each figure. In the cases of the $45^{\circ}$ and $60^{\circ}$ detectors, it can be seen that the TAE causes the pitch angle of the observed loss to increase-i.e. more deeply trapped particles are lost. This was also sometimes observed to be the case with the NBI driven Alfvén modes (see Fig. 4(a)). For the $20^{\circ}$ detector, 
the loss in the absence of the TAE was too small to detect, hence no orbit is plotted for that case. However, during the TAE, a significant loss is seen, with a typical orbit as depicted in Fig. 12(c). The intriguing feature of this data is that particles which must have originated at the ICRF resonance layer now occupy orbits which are far removed from that region. From the distance the orbit has been displaced radially from the $\mathrm{H}$ cyclotron layer in Fig. 12(c), about $0.8 \mathrm{~m}$, and the knowledge that this motion occurs in less than a fast ion slowing down time, which is $\sim 200 \mathrm{~ms}$, it is possible to infer a lower bound on the spatial diffusion coefficient for these ions. That lower bound is $\sim 3 \mathrm{~m}^{2} / \mathrm{s}$, considerably larger than spatial diffusion coefficients for fast ions observed in quiescent plasmas, which are typically of the order of $0.1 \mathrm{~m}^{2} / \mathrm{s} .[27]$

Figure 13 displays the peak pitch angle of the tail ion loss during the TAE as a function of the plasma current. For both the $45^{\circ}$ and $60^{\circ}$ detectors, the peak pitch angle diminishes as the plasma current increases. This may indicate something about the nature of the loss, but is also consistent with fact that the banana widths will be reduced as the plasma current increases, so that a particle will have to have a lower pitch angle to reach the detector position. The peak pitch angle observed in the $20^{\circ}$ detector does not change over the range observed.

Finally, Fig. 14 indicates the variation with applied ICRF power of the peak pitch angles and energies observed in the $45^{\circ}$ and $60^{\circ}$ detectors. The two detectors display contrasting behaviors: in the $45^{\circ}$ detector, the peak energy and the peak pitch angle of the particle losses are both observed to increase as the ICRF power is increased. However, the data from the $60^{\circ}$ detector reveals a constant pitch angle and energy over the entire range of ICRF power. It is unclear why the data from these two detectors, which otherwise tend to express similar trends, are so different in this instance.

\section{Discussion of H-minority ICRF Results}

The linear relationship between the TAE amplitude and loss rate suggests that the loss is due to a process which is convective in nature, rather than diffusive. Observations from the DIII-D tokamak also indicate a linear relationship between TAE amplitude and particle loss rate [13-15]. Computational models of alpha loss from CIT[23] predict loss of a convective character at first, followed by loss of a diffusive nature (loss rate quadratic in mode amplitude) as the most easily lost particles are depleted. This computation may not apply well to this experiment since the parameters used differ significantly from those in the experiment. In particular, the present experiments do not contain an isotropic distribution of fast ions, but one which is dominated by trapped ions. In addition, in the experiment, there is a continual source of new fast ions accelerated by the ICRF waves, while in the simulation there is a population which is depleted and not replenished with time.

A loss mechanism which is consistent with the observation that more deeply trapped tail ions are lost during the TAE is this: the TAE causes transport of resonant fast ions outward in major radius. Since the bounce frequency of the particles is roughly equal to the TAE frequency, this is possible. The $20^{\circ}$ detector data seems especially to support this interpretation, since the tail ions appear radially outward from where they are created, and are moved a substantial distance (several banana widths) to the detector within a fraction of a slowing down time. Such a mechanism might also explain the observed linear relationship between loss rate and mode amplitude. A similar mechanism was previously found to be responsible for the expulsion of neutral beam ions during fishbone oscillations [28].

\section{Summary}


In summary, Alfvén modes in TFTR driven both by NBI ions and by H-minority ICRF tail ions produce fast ion losses. In both cases, enhanced losses of fast ions are seen coincident with the instability. In the neutral beam case, the loss of trapped fast ions doubles but still appears to be a negligible fraction of the input power. In the ICRF tail ion driven TAE, the loss of applied plasma heating power due to the mode can be quite large: up to $\sim 10 \%$ of the input power. In both cases, the data is consistent with a power loss which varies linearly with the mode amplitude. In the ICRF driven TAEs, more deeply trapped particles are lost during the mode. This shift in pitch angles is consistent with the outward major radial transport of mode-resonant ions.

Further work in the area of TAE-induced fast ion losses could include calculation of particle losses from TFTR plasmas, with a focus on identifying the classes of particles lost and the processes which result in the observed characteristics of the loss reported here. Additional experimental work with diagnostics of confined fast ions could be used to verify accuracy of the models for the driving terms for both passing and trapped particles, as well as the strength of the "sideband" drive at $v_{f} \sim v_{A} / 3$. The issue of alpha particle driven TAEs and resulting alpha losses is being studied in TFTR's DT experiments.

\section{Acknowledgements}

This work was supported by US DoE contract DE-AC02-76-CHO-3073.

\section{References}

[1] PUTVINSKI, S., BERK, H., BARABASCHI, P., et al., in Proc. of the 15th International Conference on Plasma Physics and Controlled Nuclear Fusion Research, Seville, 1994, (International Atomic Energy Agency, Vienna, 1995), IAEA-CN-60, paper E-P-4.

[2] ZWEBEN, S. J., Nucl. Fusion 29 (1989) 825.

[3] ZWEBEN, S. J., et al., Nucl. Fusion 30 (1990) 1551.

[4] ZWEBEN, S. J., et al., Phys. Fluids B 2 (1990) 1411.

[5] BOIVIN, R. L., "Measurements of Charged Fusion Product Diffusion in TFTR," Ph.D. Thesis, Princeton University, October 1991.

[6] ZWEBEN, S. J.s DARROW, D. S., HERRMANN, H. W., et al., Nucl. Fusion 35 (1995) 893.

[7] TOBITA, K., et al., Nucl. Fusion 34 (1994) 1097.

[8] WONG, K.-L., et al., Phys. Rev. Lett. 66 (1991) 1874.

[9] WONG, K.-L.,et al., Phys. Fluids B 4 (1992) 2122.

[10] WILSON, J. R., et al., in Proc. of the 14th International Conference on Plasma Physics and Controlled Nuclear Fusion Research, Wurzburg, Germany, October 1992, paper IAEA-CN-56/E-2-2. 
J. Y. Park and S. Z. Zweben, Princeton Plasma Physics Laboratory Report number PPPL-2971 (1994).

[11] BOIVIN, R. L., KILPATRICK, S., MANOS, D., and ZWEBEN, S. J., Rev. Sci. Instrum. 61 (1990) 3208.

[12] DARROW, D. S., HERRMANN, H. W., JOHNSON, D. W., et al., Rev. Sci. Instrum. 66 (1995) 476.

[13] DUONG, H., HEIDBRINK, W. W., STRAIT, E. J., et al., Nucl. Fusion 33 (1993) 749.

[14] HEIDBRINK, W. W., et al., Nucl. Fusion 31 (1991) 1635.

[15] STRAIT, E. J., et al., Nucl. Fusion 33 (1993) 1849.

[16] DARROW, D. S., et al., Proc. 19th EPS Conf. on Controlled Fusion and Plasma Physics, Part I (1992) 431 .

[17] SESNIC, S., et al., Nucl. Fusion 33 (1993) 1877.

[18] KIMURA, H., SAIGUSA, M., MORIYAMA, S., et al., Phys. Lett. A 199 (1995) 86.

[19] SAIGUSA, M., KIMURA, H., MORIYAMA, S., et al., Plasma Phys. Control. Fusion 37 (1995) 295.

[20] KIMURA, H., SAIGUSA, M., KONDOH, T., et al., Jap. J. Plasma Fusion Res. 71 (1995) 1147.

[21] ALI-ARSHAD, S. and CAMPBELL, D. J., Plasma Phys. Control. Fusion 37 (1995) 715 .

[22] FASOLI, A., et al., Report Number JET-P(95)30, submitted to Nucl. Fusion.

[23] SIGMAR, D. J., et al., Phys. Fluids B 4 (1992) 1506.

[24] GOLDSTON, R. J., WHITE, R. B., and BOOZER, A. H., Phys. Rev. Lett. 47 (1981) 647.

[25] HEIDBRINK, W. W., DUONG, H. H., MANSON, J., WILFRID, E., OBERMAN, C., and STRAIT, E. J., Phys. Fluids B 5 (1993) 2176.

[26] PHILLIPS, C. K., WILSON, J. R., BELL, M., et al., in Proceedings of the 10th Topical Conference on Radio Frequency Power in Plasmas , (Boston 1993), American Institute of Physics, New York (1994) 44.

[27] HEIDBRINK, W. W. and SADLER, G. J., Nucl. Fusion 34 (1994) 535.

[28] WHITE, R. B., et al., Phys. Fluids 26 (1983) 2958. 


\section{Figure Captions}

Figure 1: Time history of the Mirnov coil signal, the neutron rate, and the rate of beam ion loss for a plasma in which an Alfvén mode was driven unstable by $100 \mathrm{keV}$ deuterons from the neutral beam heating system of TFTR.

Figure 2: Frequency spectra of the signal from a Mirnov coil during and between bursts of mode activity in the shot shown in Fig. 1.

Figure 3: (a) Fractional change in the neutron rate plotted against the time integral of the rms Alfvén mode amplitude. The data are from bursts which occurred in twelve nominally identical discharges. The magnitude of the drop in the neutron rate is computed as in Ref. 10, by extrapolating the neutron signal at constant slope past the burst, and measuring the difference between this extrapolated level and the minimum rate reached just after the burst. This is then divided by the average neutron rate to give the fractional change. The integral Alfvén mode amplitude is determined by high pass filtering the digitized Mirnov coil signal to retain $\mathrm{f}>62.5 \mathrm{kHz}$ only, integrating the signal in time to restore $\delta \mathrm{B}$ from $\mathrm{d}(\delta \mathrm{B}) / \mathrm{dt}$, then computing the rms amplitude of the magnetic fluctuations, subtracting a background level due to turbulence, and finally integrating the result in time. (b) Total loss to the midplane probe, integrated over the duration of the burst, plotted against the time integral of the rms Alfvén mode amplitude. (c) Fractional change in the neutron rate plotted against the time integral of the rms magnetic activity between 10 and $62.5 \mathrm{kHz}$.

Figure 4: (a) Detected pitch angle distributions of beam ion losses during and between bursts of TAE activity. The heights of the curves are rescaled to be equal for ease of comparison. A small increase in pitch angle is observed during the Alfvén mode burst, although during other bursts the pitch angle is sometimes seen to decrease. The boundary between passing and trapped particles for this discharge is at a pitch angle of $47^{\circ}$. (b) Detected distributions of gyroradii from the same times as in part (a). The loss appears to shift very slightly to lower gyroradii during the mode. Again, the heights have been rescaled.

Figure 5: Orbits computed from the observed pitch angles at the $20^{\circ}$ detector at several different positions. All are banana orbits with their tips within the stochastic ripple domain, which is the darker shaded region in the plasma cross-section. The probe aperture positions were: $60718, R=3.49 \mathrm{~m} ; 60721$, $\mathrm{R}=3.435 \mathrm{~m}$; and $60722, \mathrm{R}=3.41 \mathrm{~m}$.

Figure 6: Total beam ion loss to the $20^{\circ}$ probe versus probe position. The limiter is at $\mathrm{R}=352.5 \mathrm{~cm}$ at this poloidal angle. The data shows roughly an exponential scrape-off, with a characteristic length of $\sim 6 \mathrm{~cm}$.

Figure 7: The observed peak pitch angle and peak gyroradius at the $20^{\circ}$ detector versus probe position, for Alfvén mode driven neutral beam ion loss.

Figure 8: Frequency spectrum of Mirnov coil data during an ICRF H-minority tail driven TAE mode.

Figure 9: Time history of a discharge with $\mathrm{H}$-minority ICRF tail ion driven TAE. (a) Tail ion loss rate at the $45^{\circ}$ escaping alpha detector. (b) TAE amplitude from Mirnov coil. (c) Soft X-ray signal showing the sawtooth behavior of the discharge. (d) Applied ICRF power. 
Figure 10: (a) TAE amplitude and escaping tail ion loss power versus applied ICRF power. (b) Escaping tail ion loss power and density fluctuation level as measured by a microwave reflectometer versus the mode amplitude measured by the Mirnov coils.

Figure 11: TAE-induced loss of tail ions versus poloidal angle.

Figure 12: (a) Escaping ICRF tail ion orbits with and without the TAE, as seen at the $60^{\circ}$ detector. (b) Escaping ICRF tail ion orbits with and without the TAE, as seen at the $45^{\circ}$ detector. (c) Escaping ICRF tail ion orbits during the TAE only, as seen at the $20^{\circ}$ detector.

Figure 13: Peak pitch angle of the TAE-induced tail ion loss in several detectors as a function of plasma current.

Figure 14: (a) Peak pitch angle of the TAE-induced H-minority tail ion loss in the $45^{\circ}$ detector plotted versus applied ICRF power. (b) Peak energy of the TAE-induced H-minority tail ion loss in the $45^{\circ}$ detector plotted versus applied ICRF power. (c) Peak pitch angle of the TAE-induced H-minority tail ion loss in the $60^{\circ}$ detector plotted versus applied ICRF power. (d) Peak energy of the TAE-induced Hminority tail ion loss in the $60^{\circ}$ detector plotted versus applied ICRF power. 


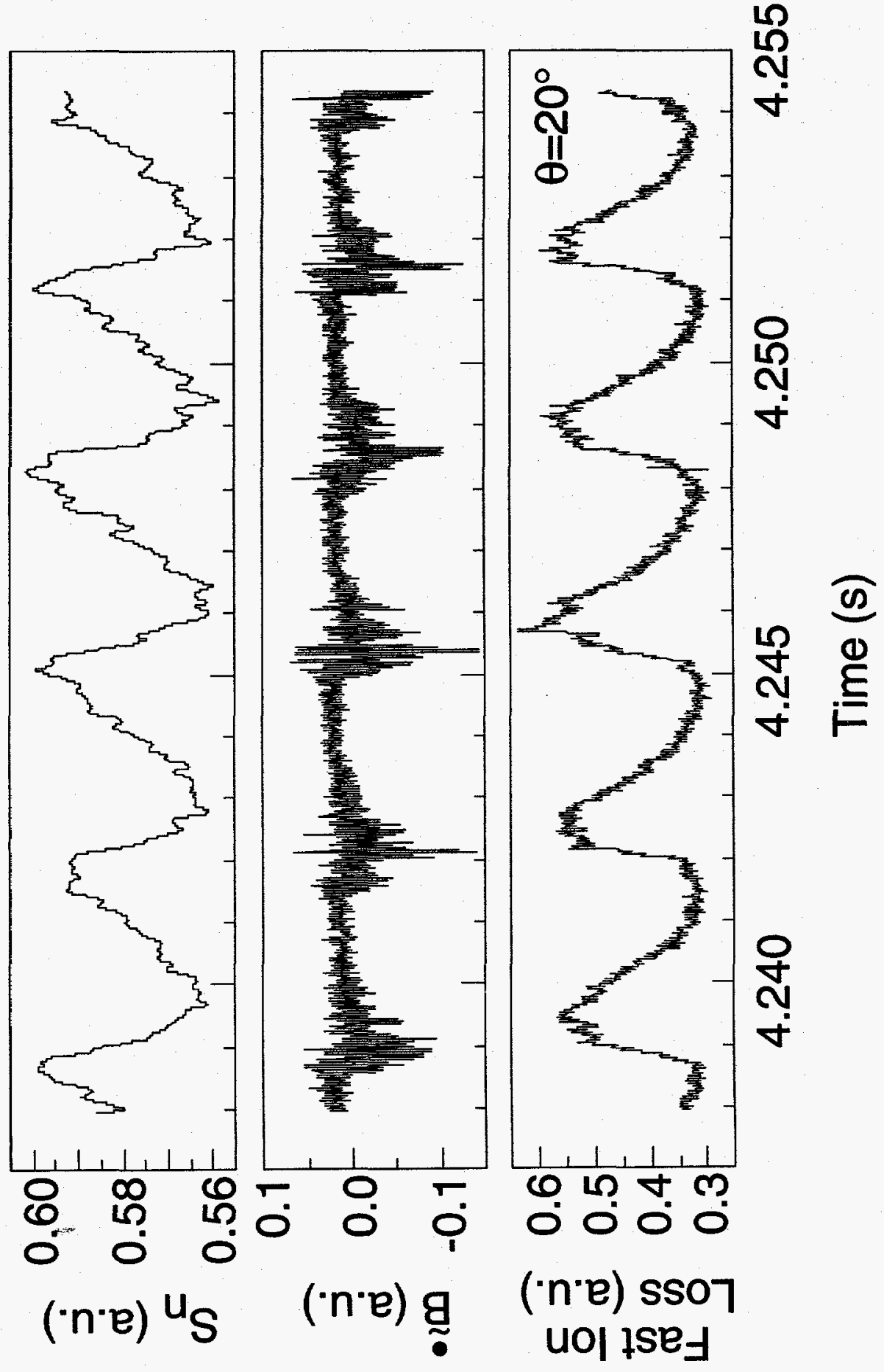

Figure 1 

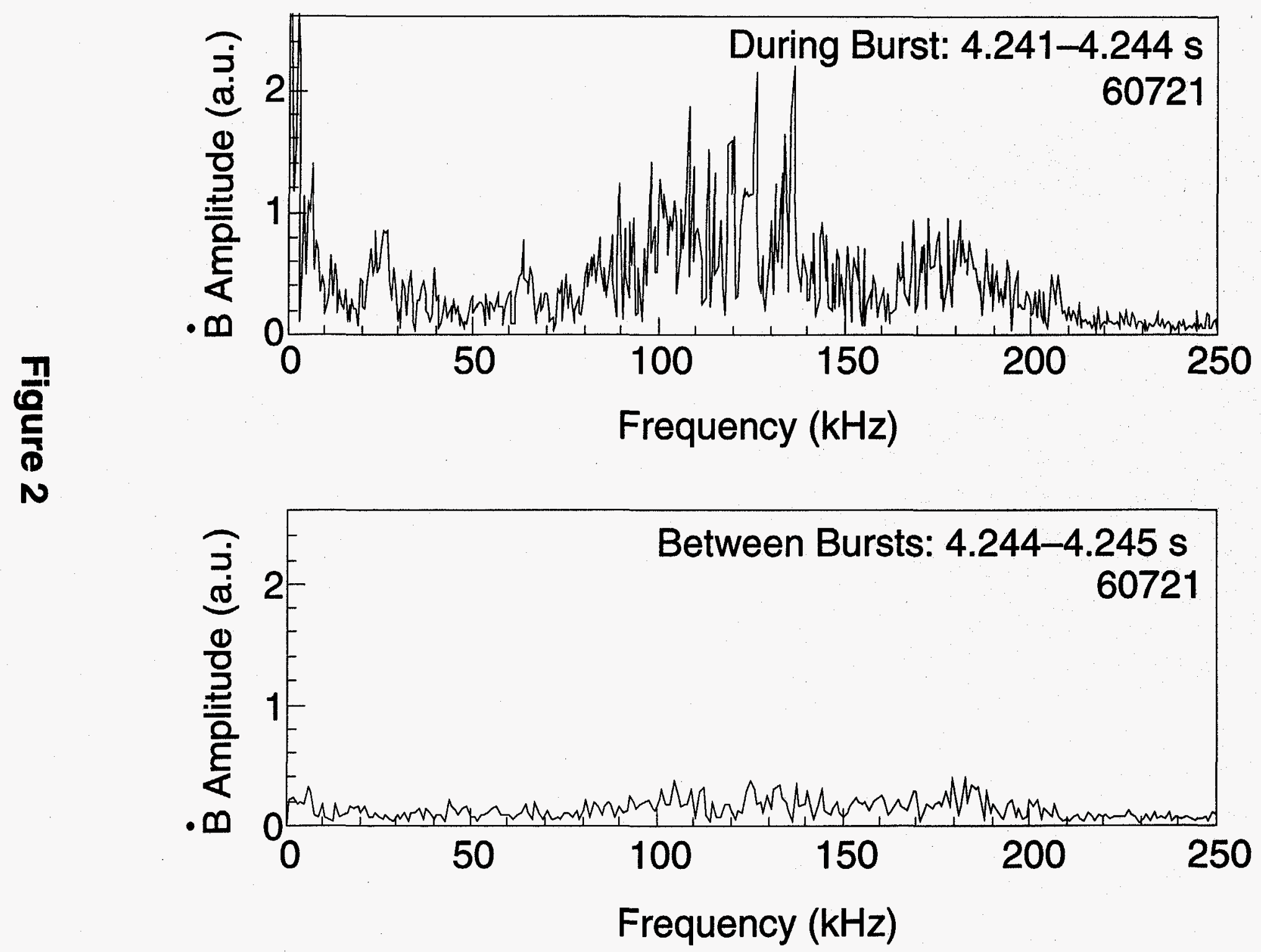


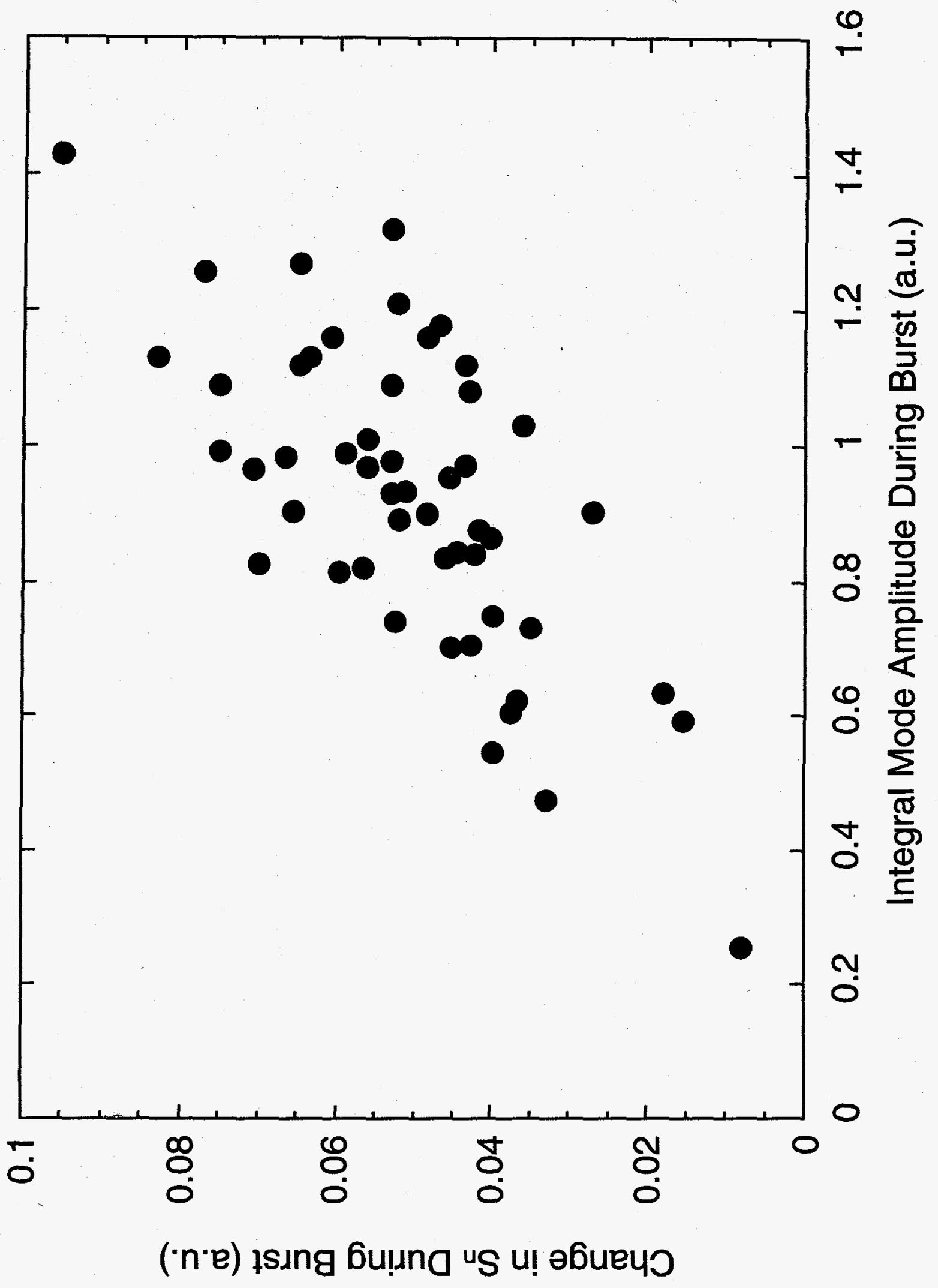

Figure 3a 


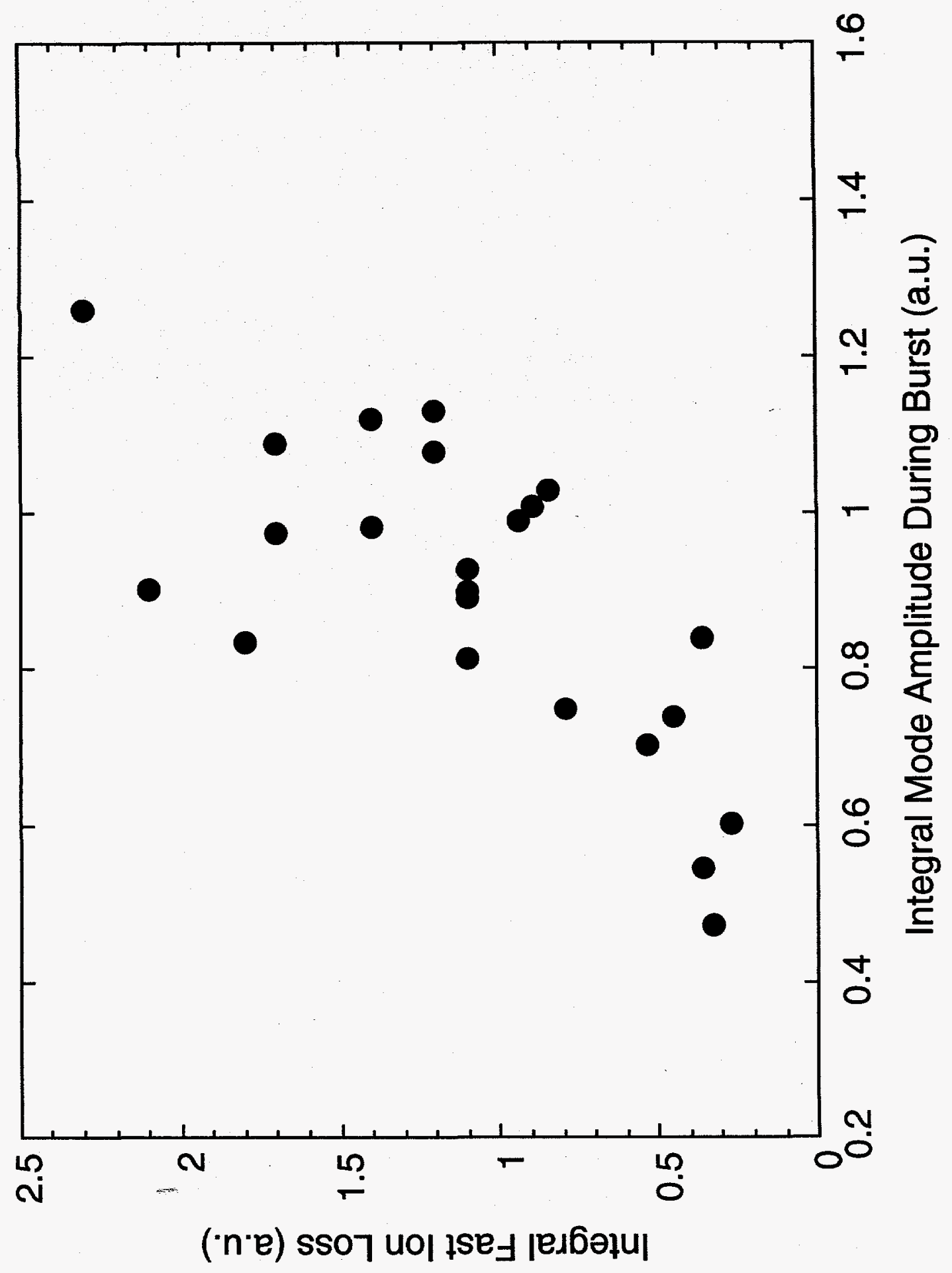

Figure $\mathbf{3 b}$ 


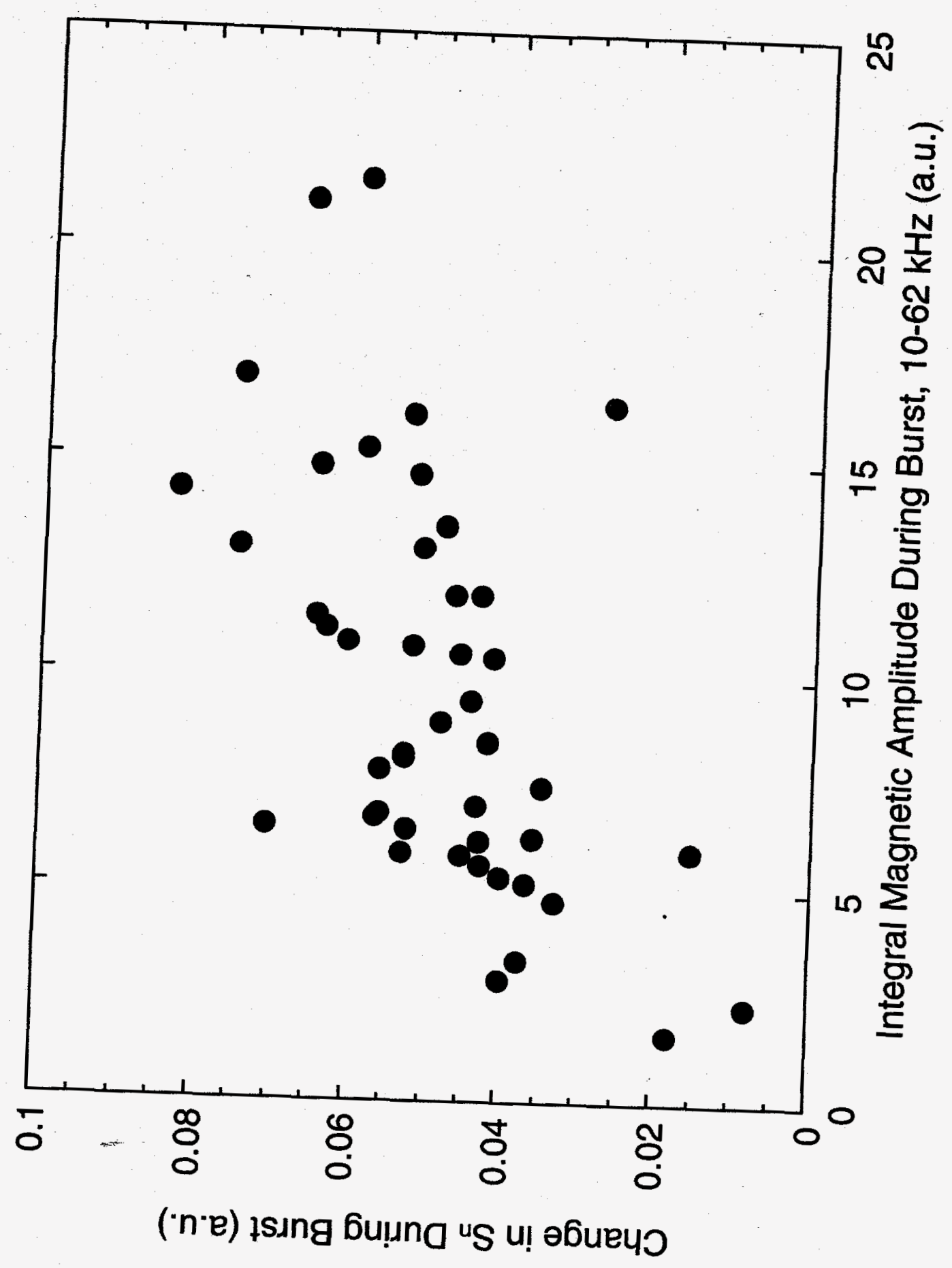

Figure 3c 


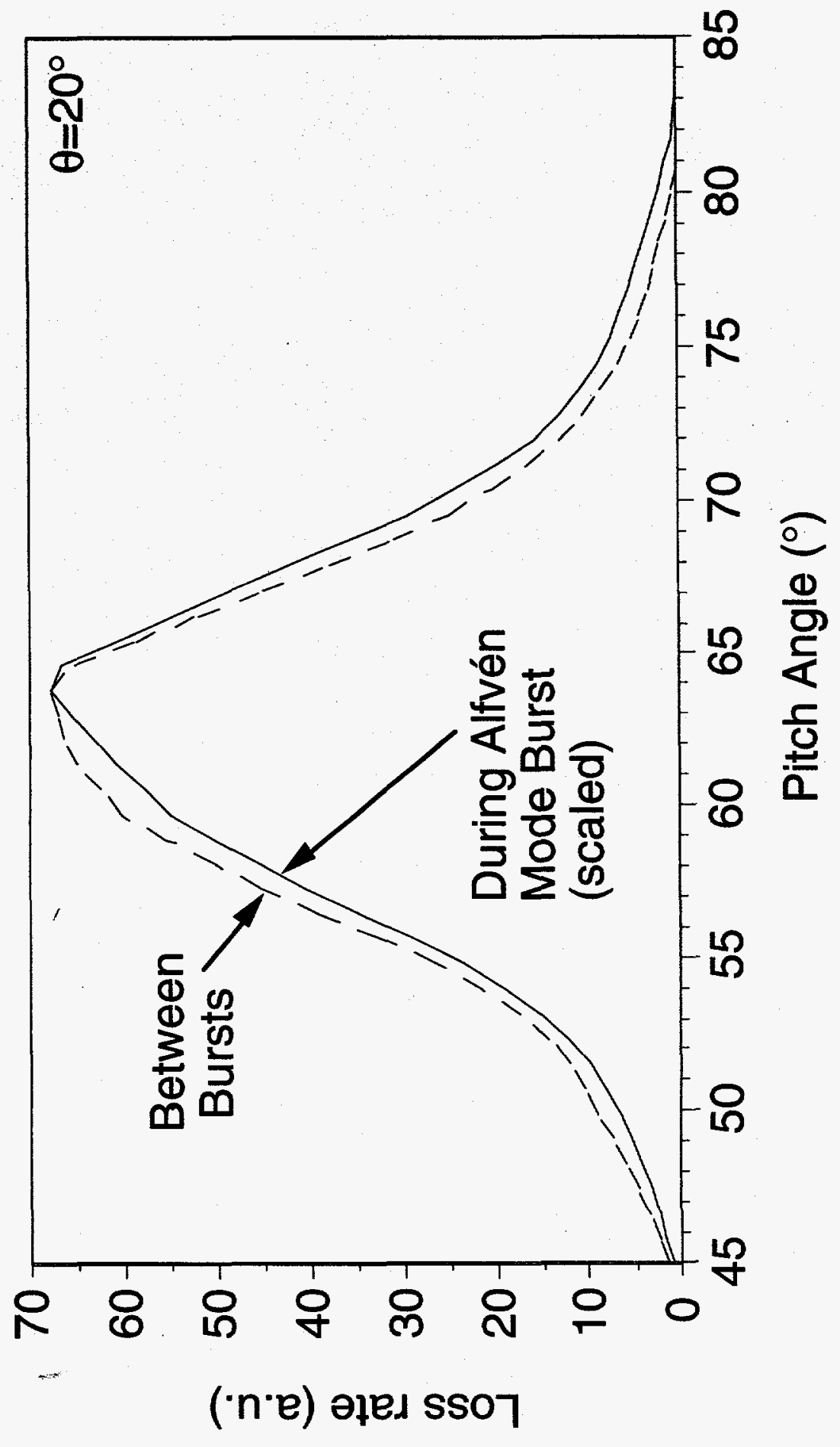

Figure 4a 


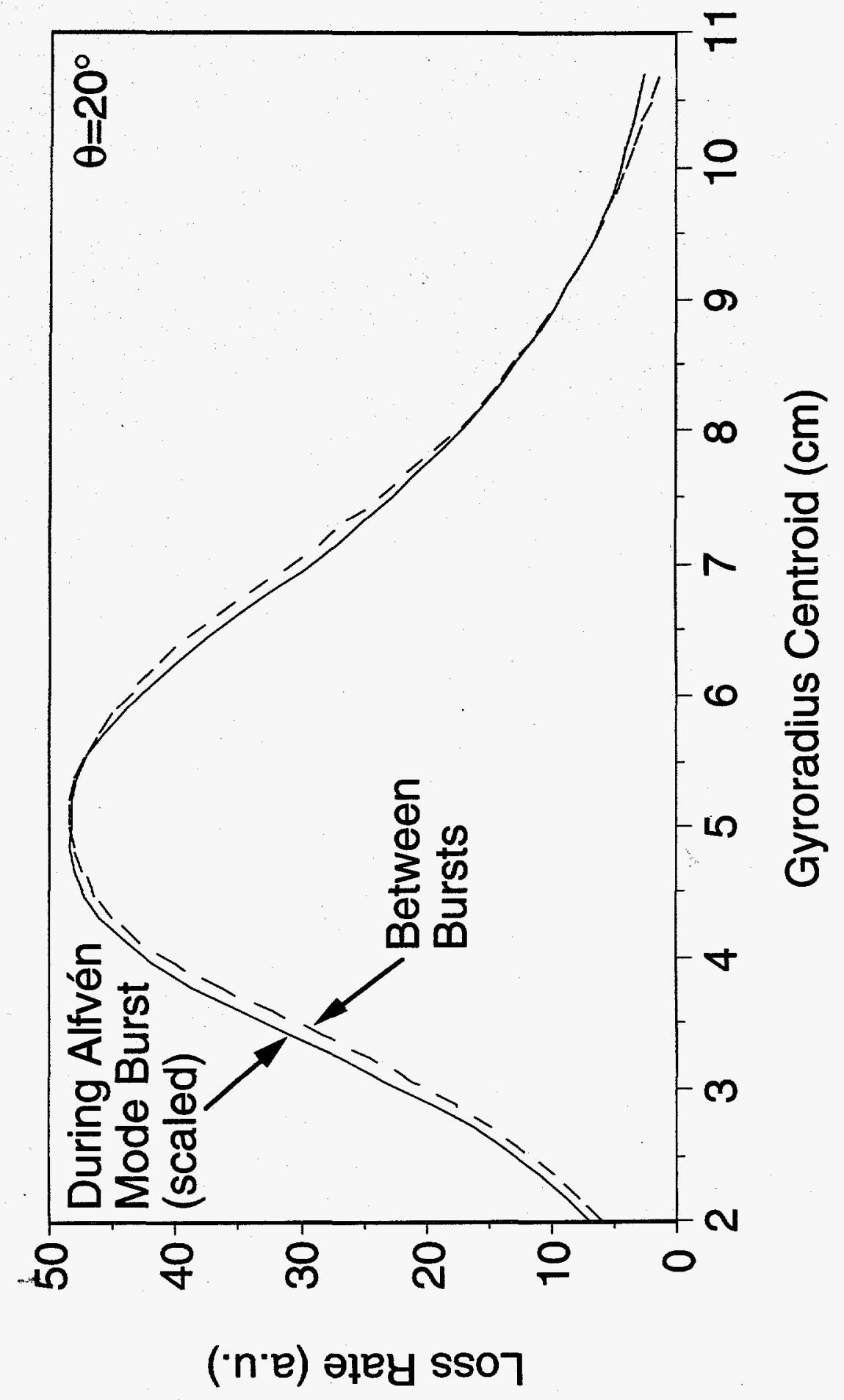

Figure 4b 


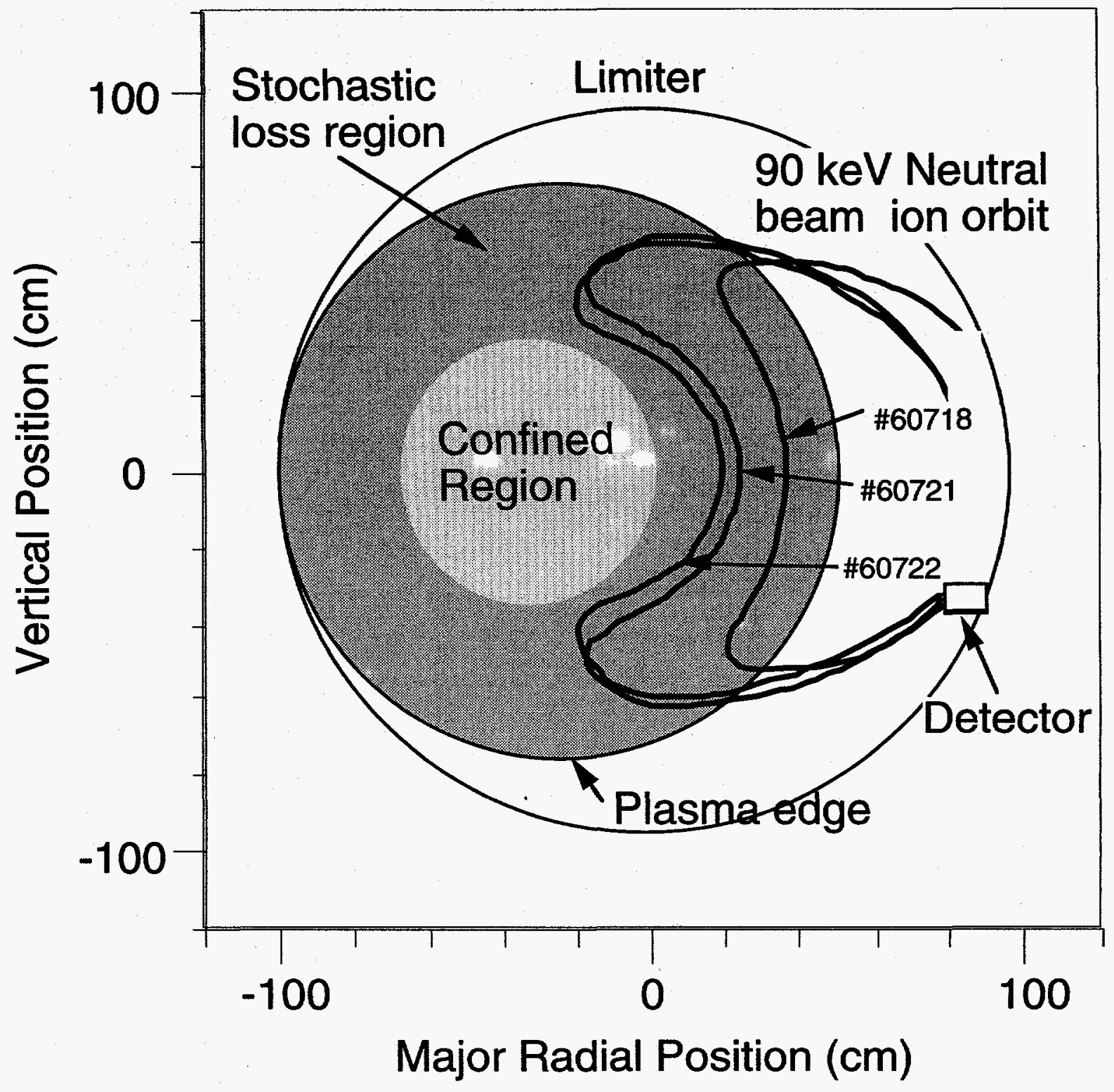

Figure 5 


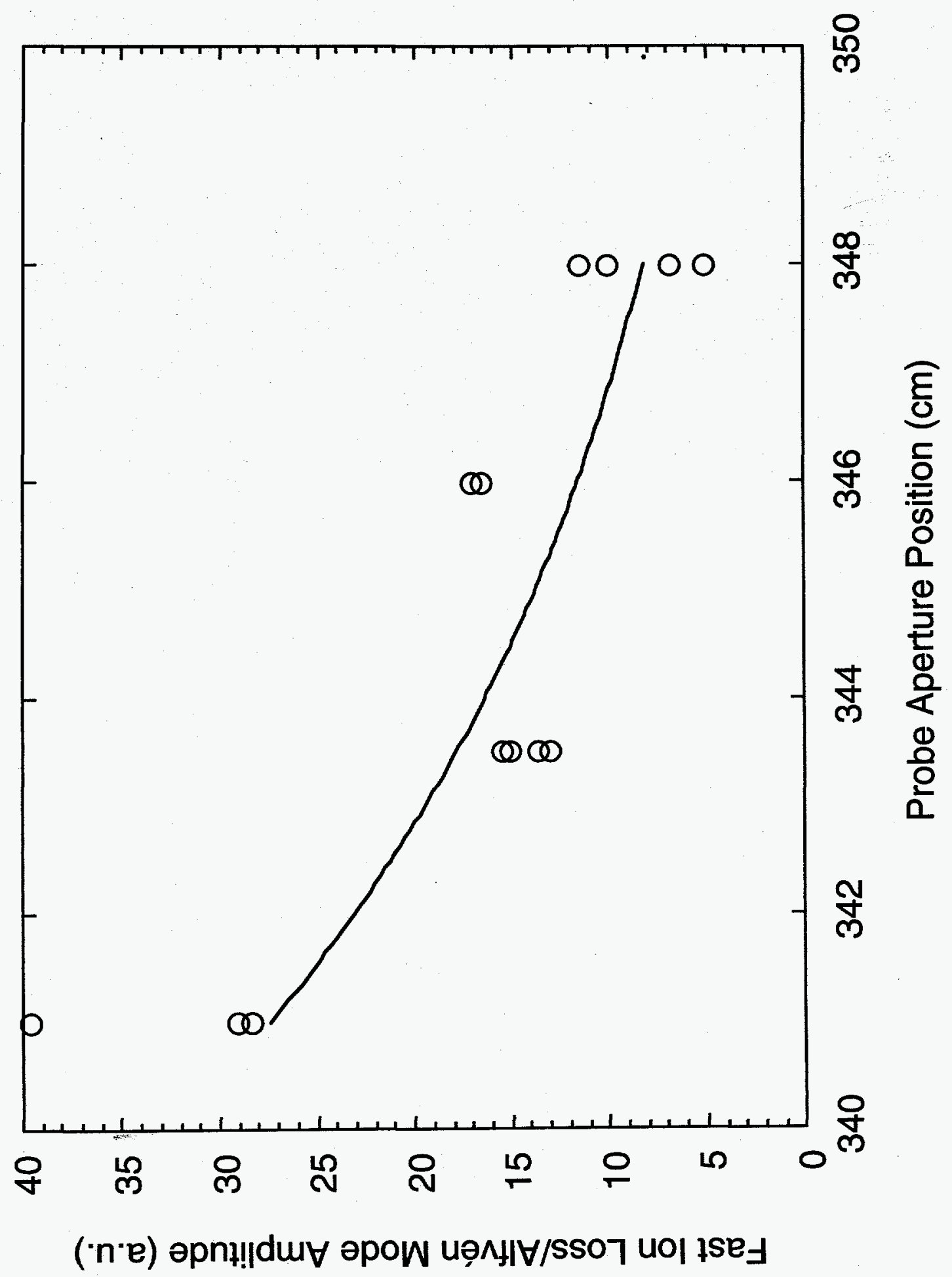

Figure 6 


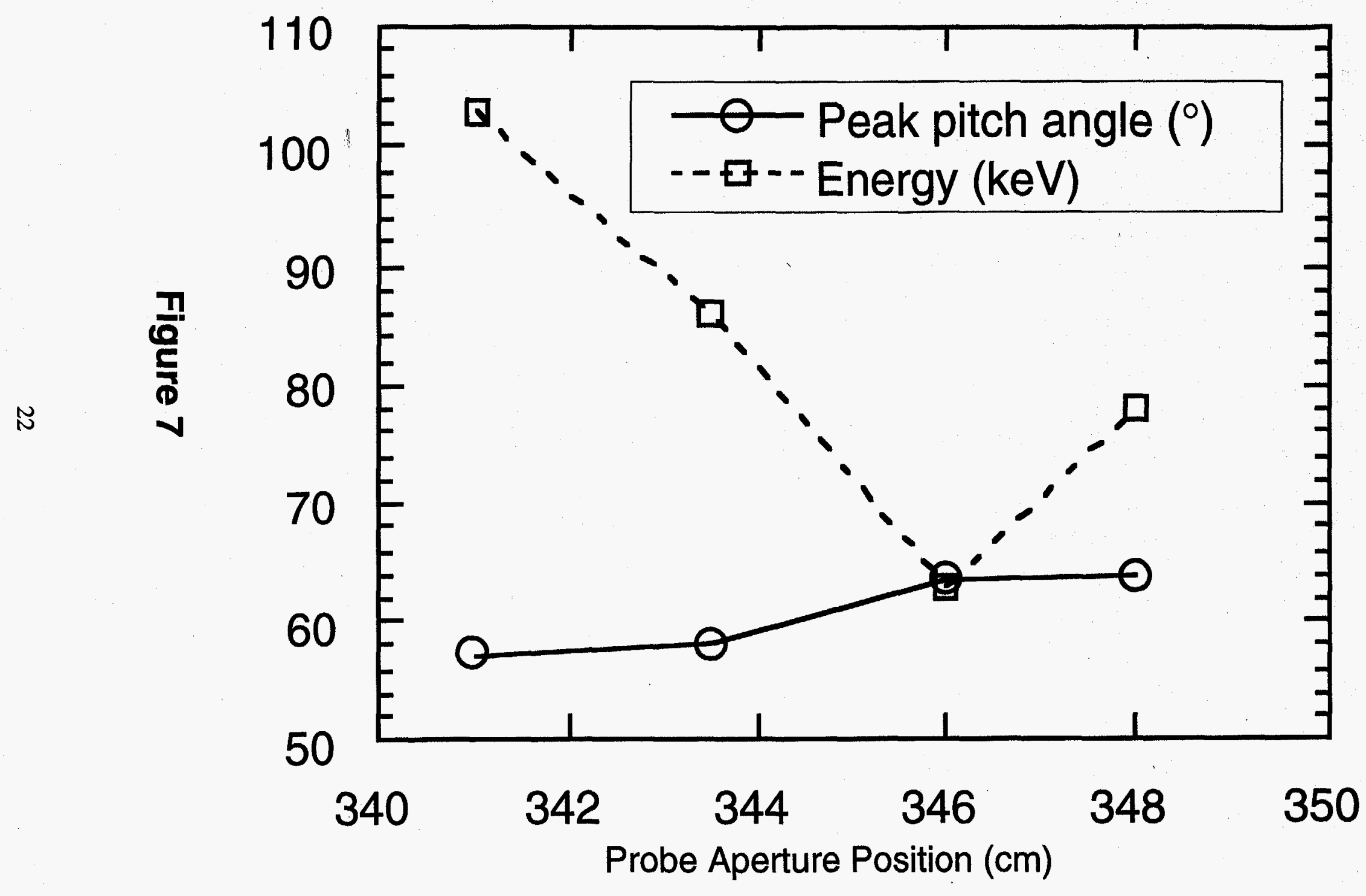




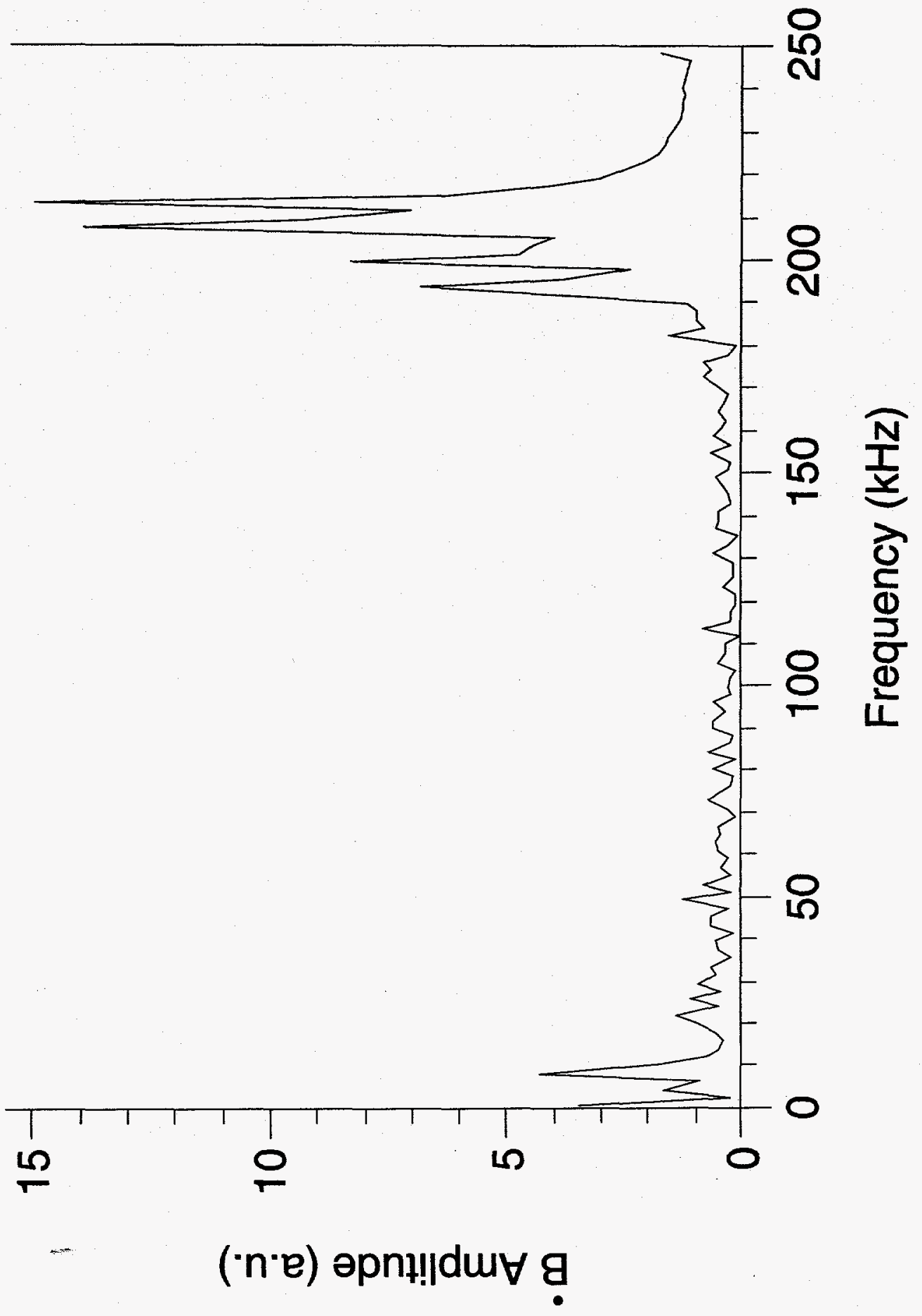

Figure 8 
TAE Ampl. Tail ion loss

ICRF Power (MW)

- $150-200 \mathrm{kHz} \quad 45^{\circ}$ detector

N

$\frac{\pi}{6}$
$\frac{1}{0}$
0

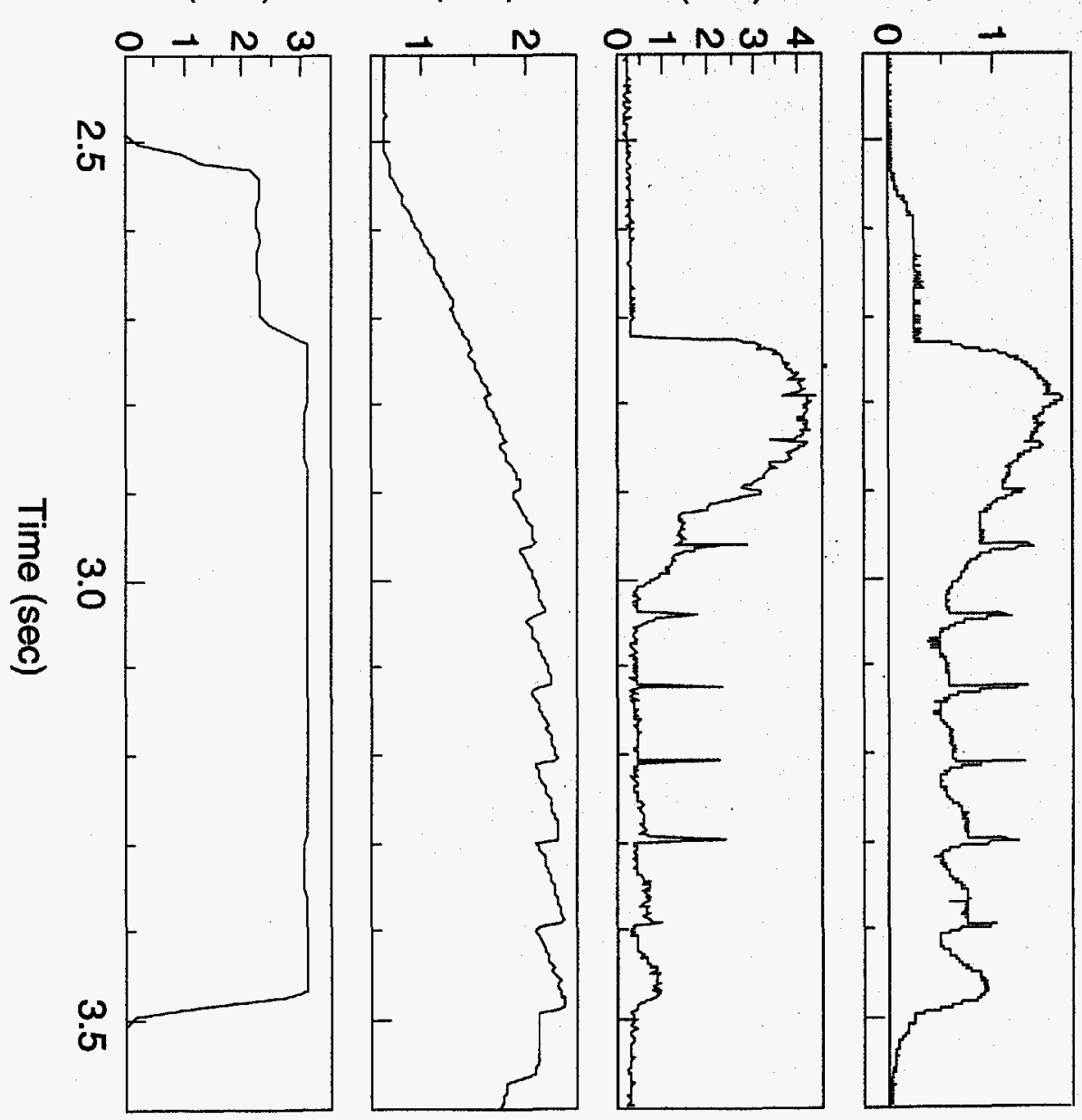


- 0 - Escaping tail ion power loss (\% of input)

口 Mode Amplitude (a.u.)

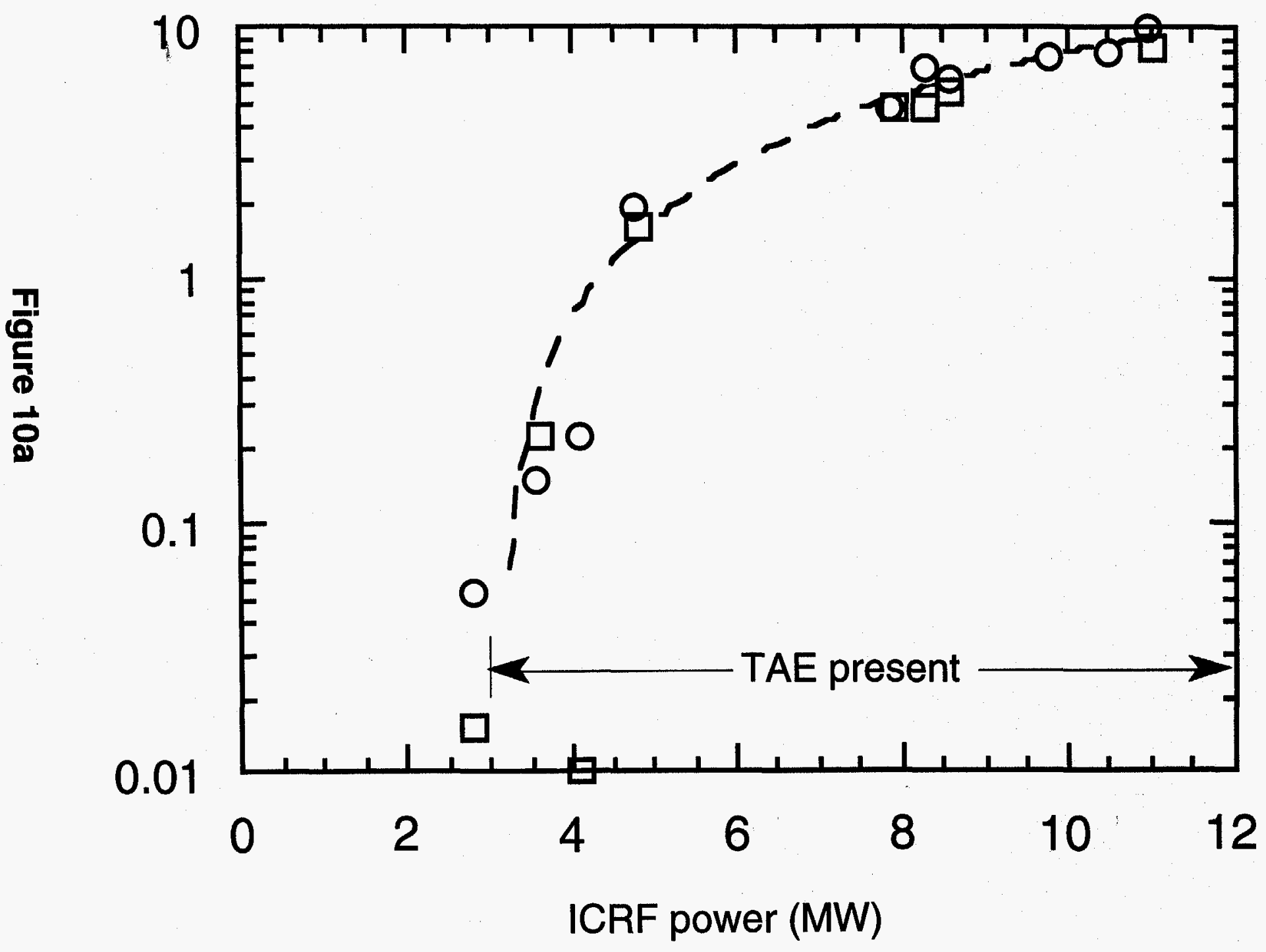




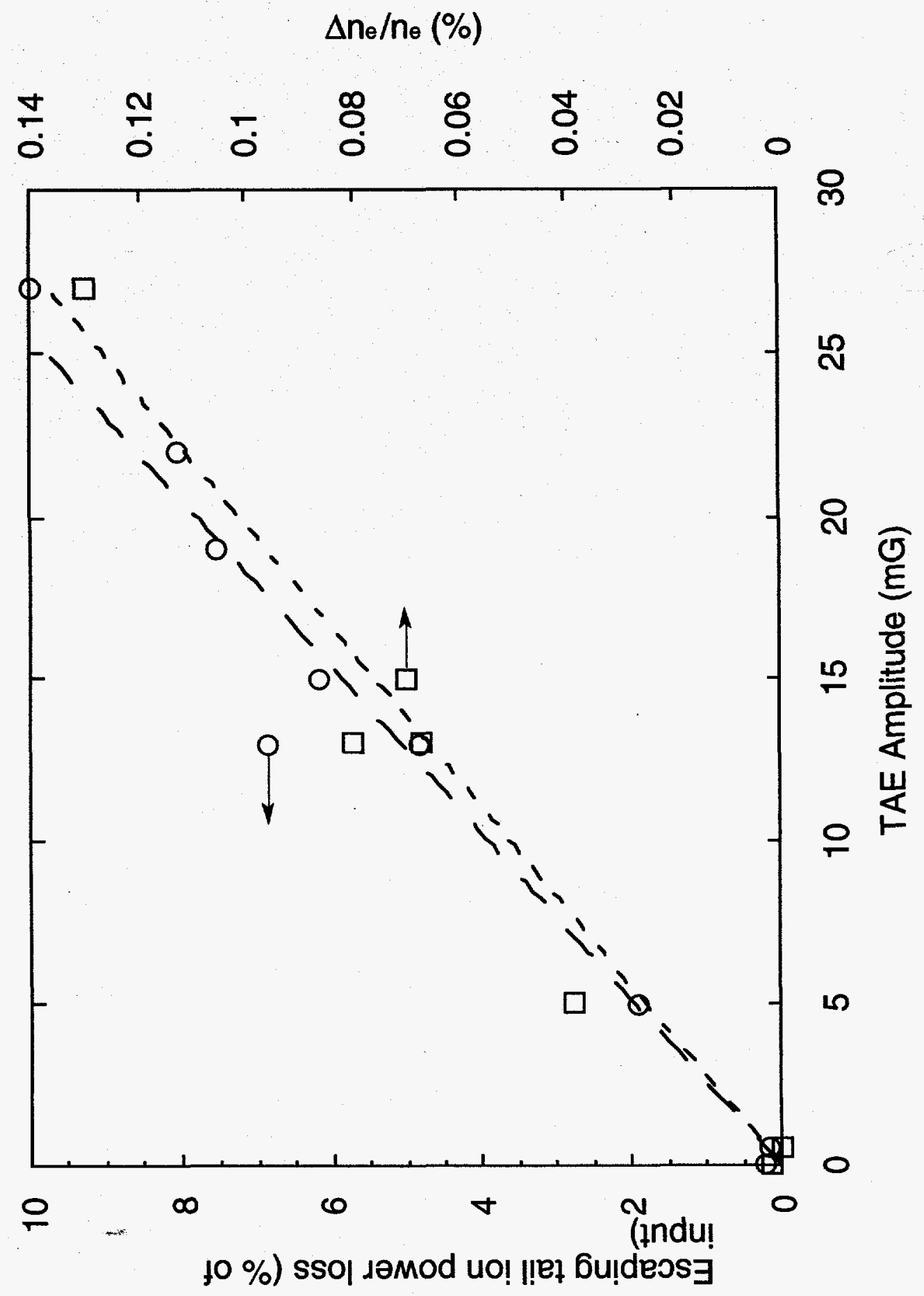

Figure 10b 


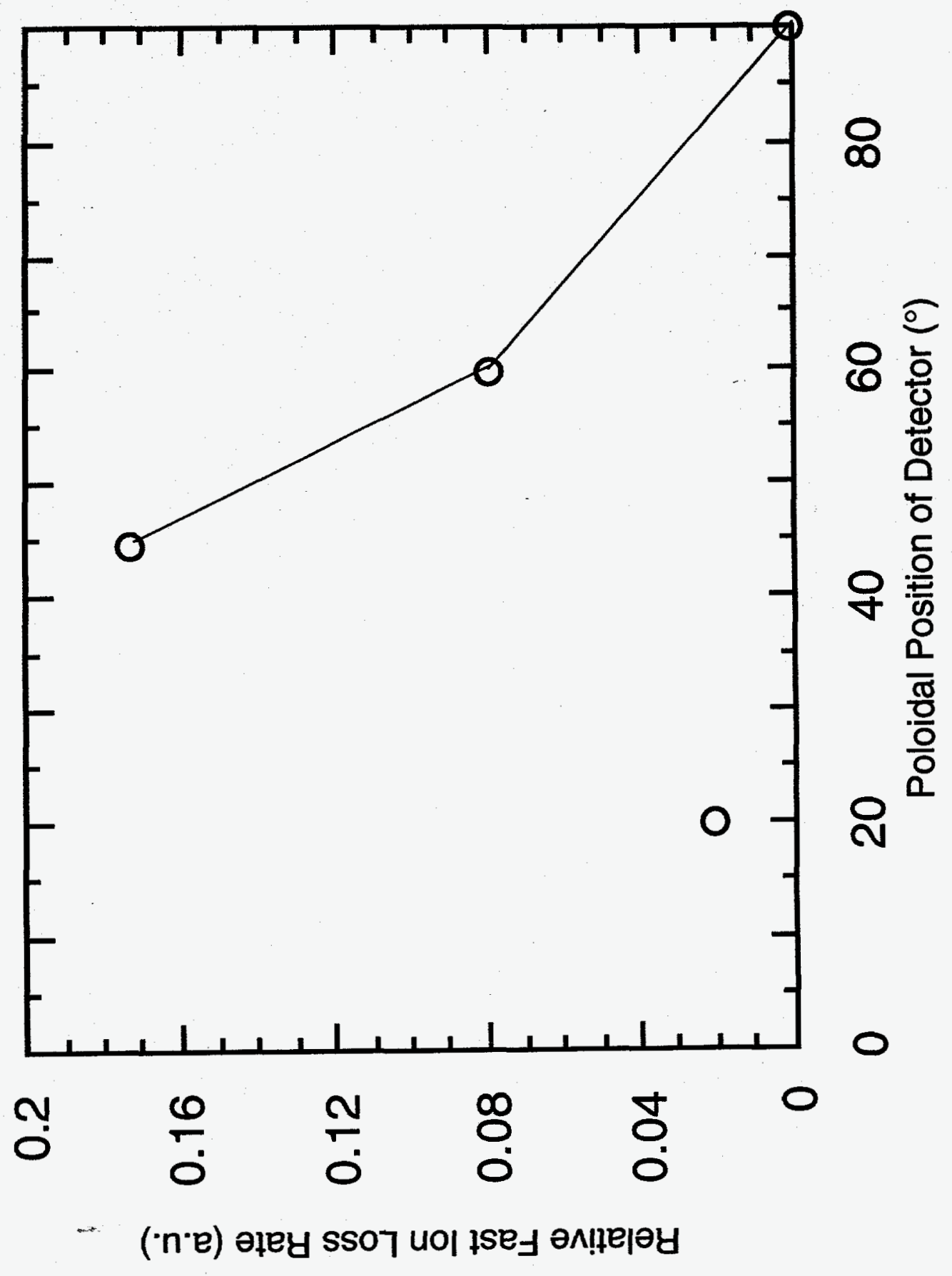

Figure 11 

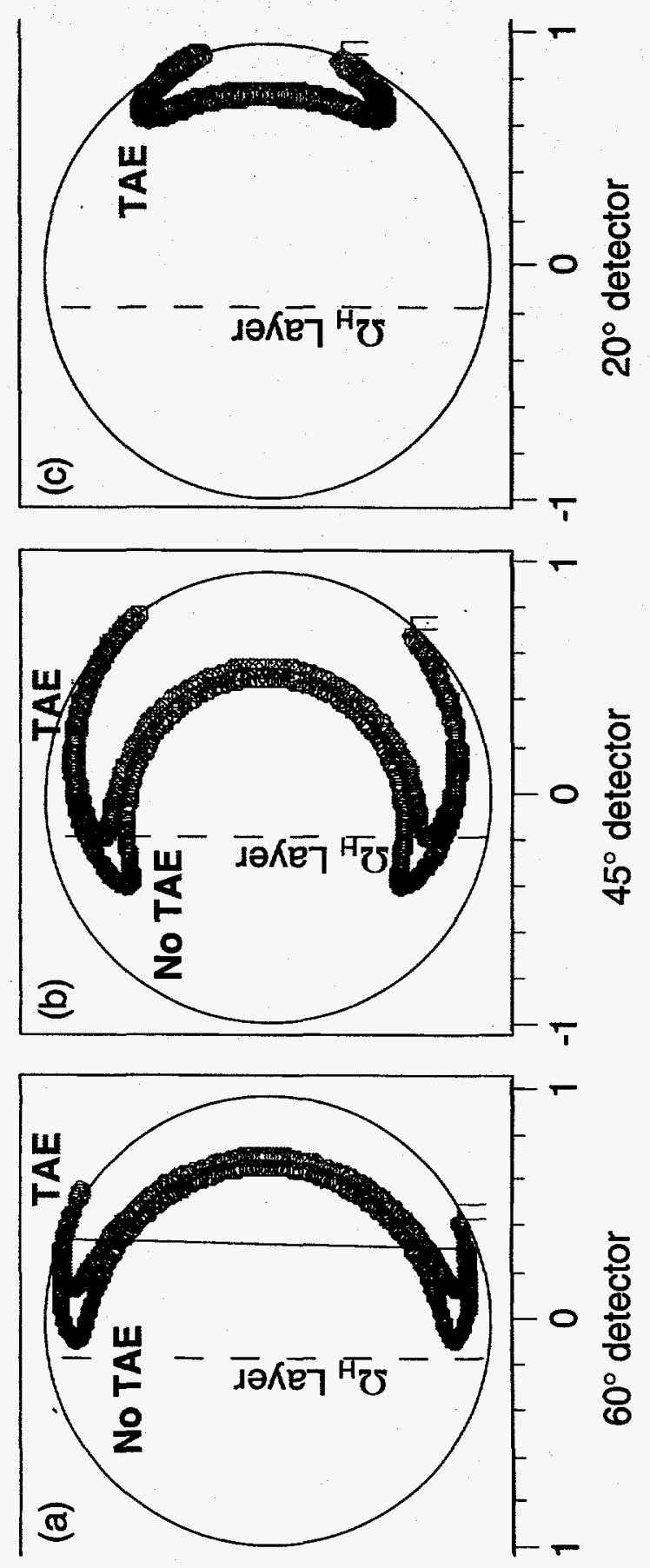

Figure 12 


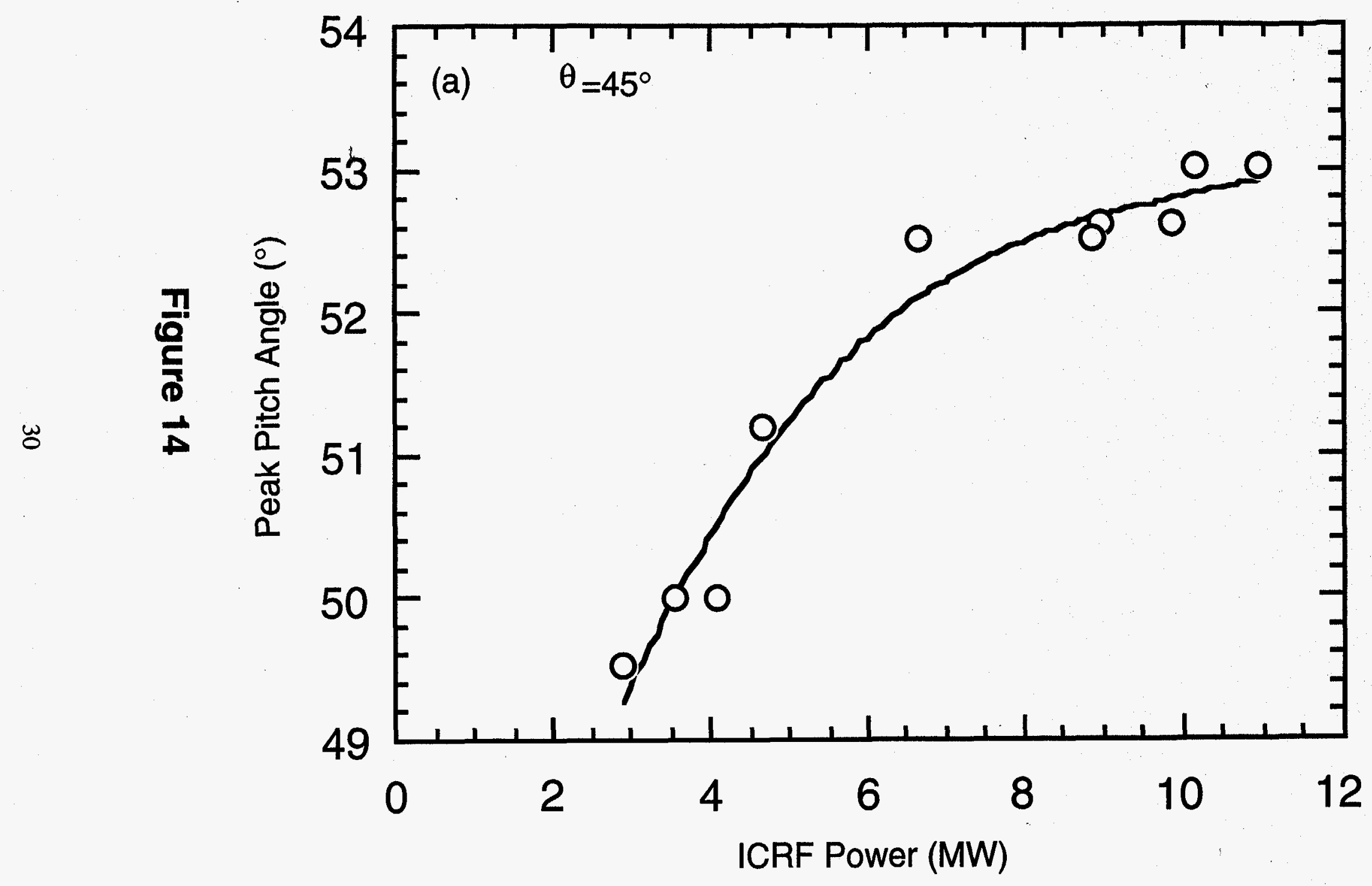




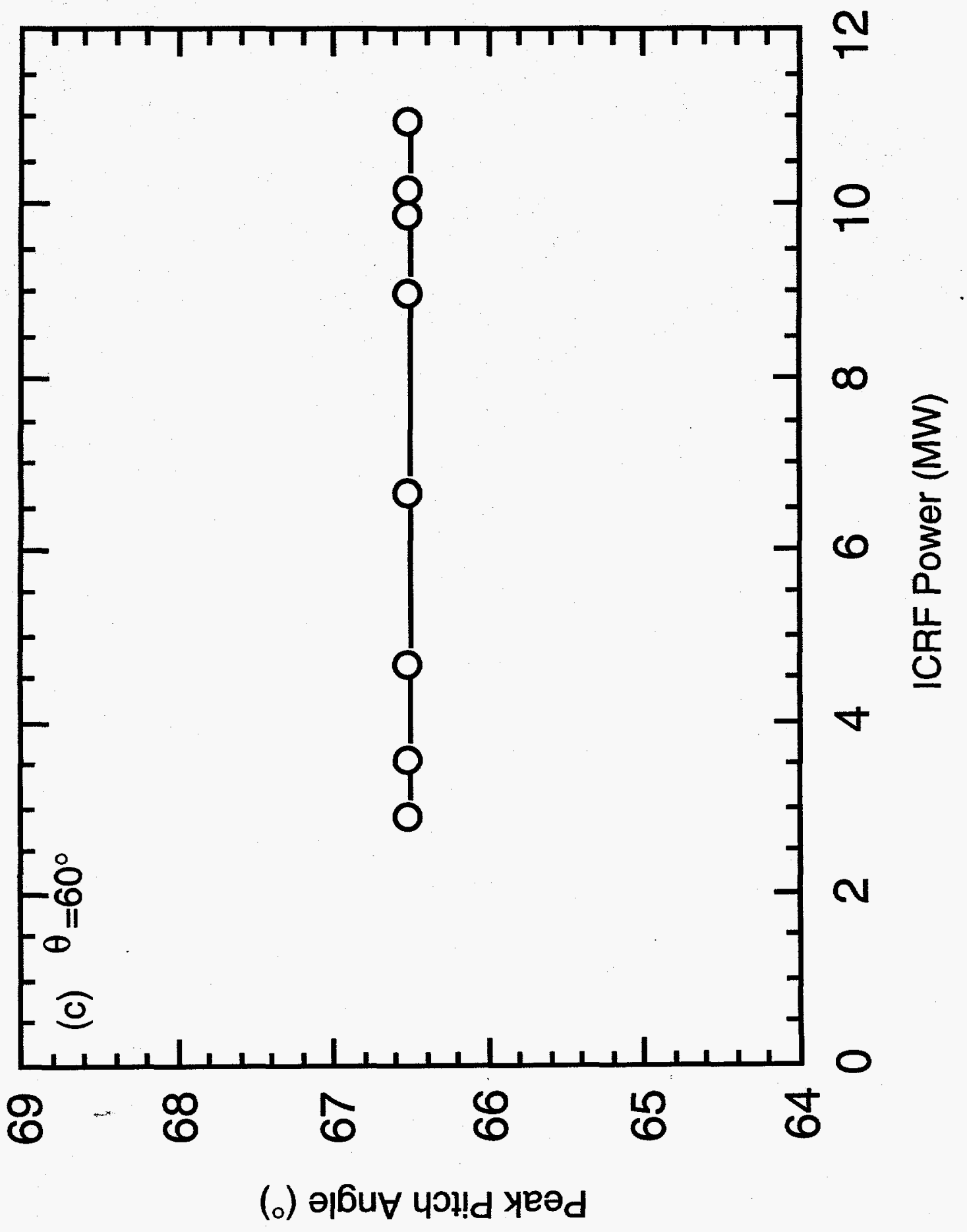

Figure 14 


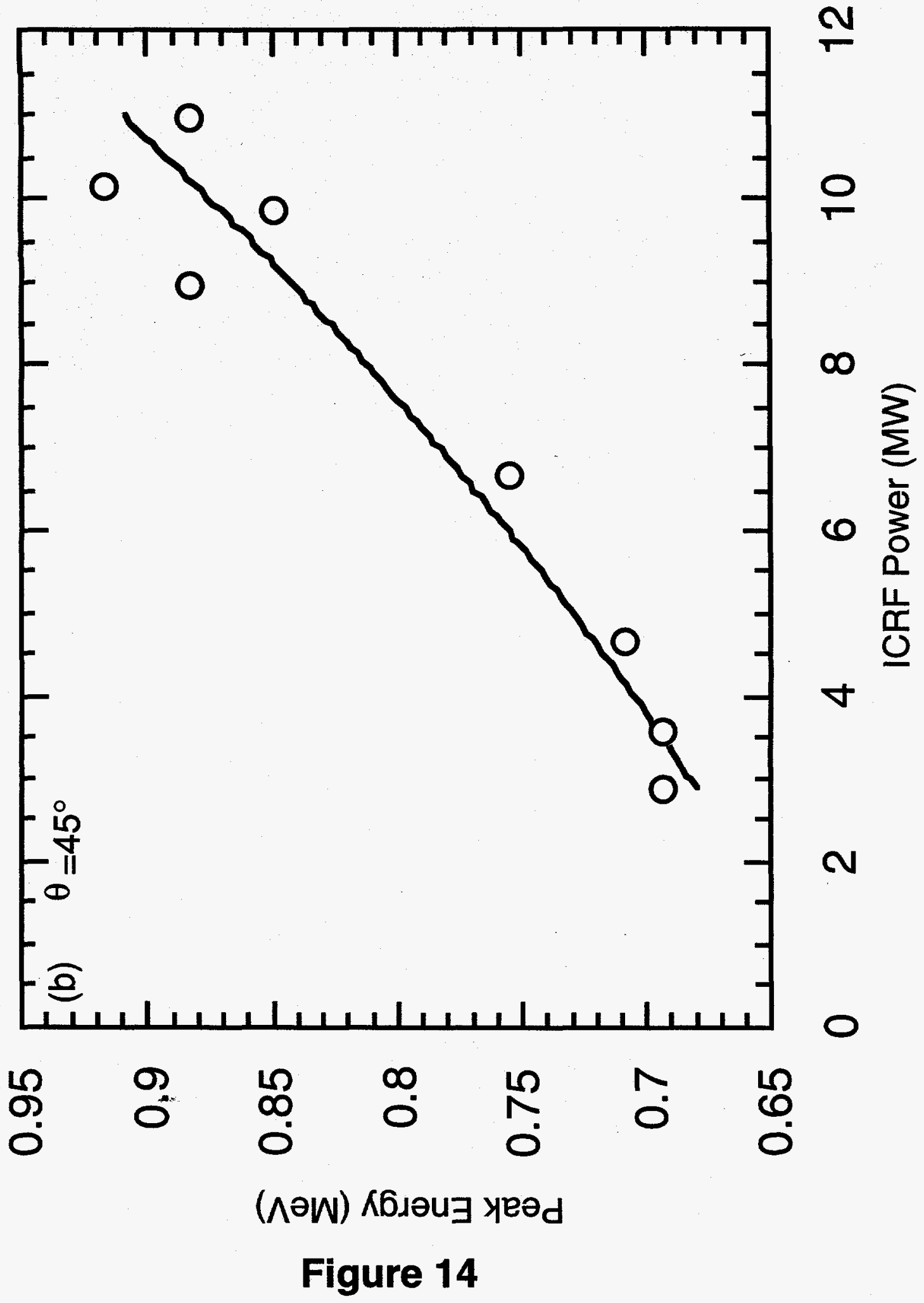




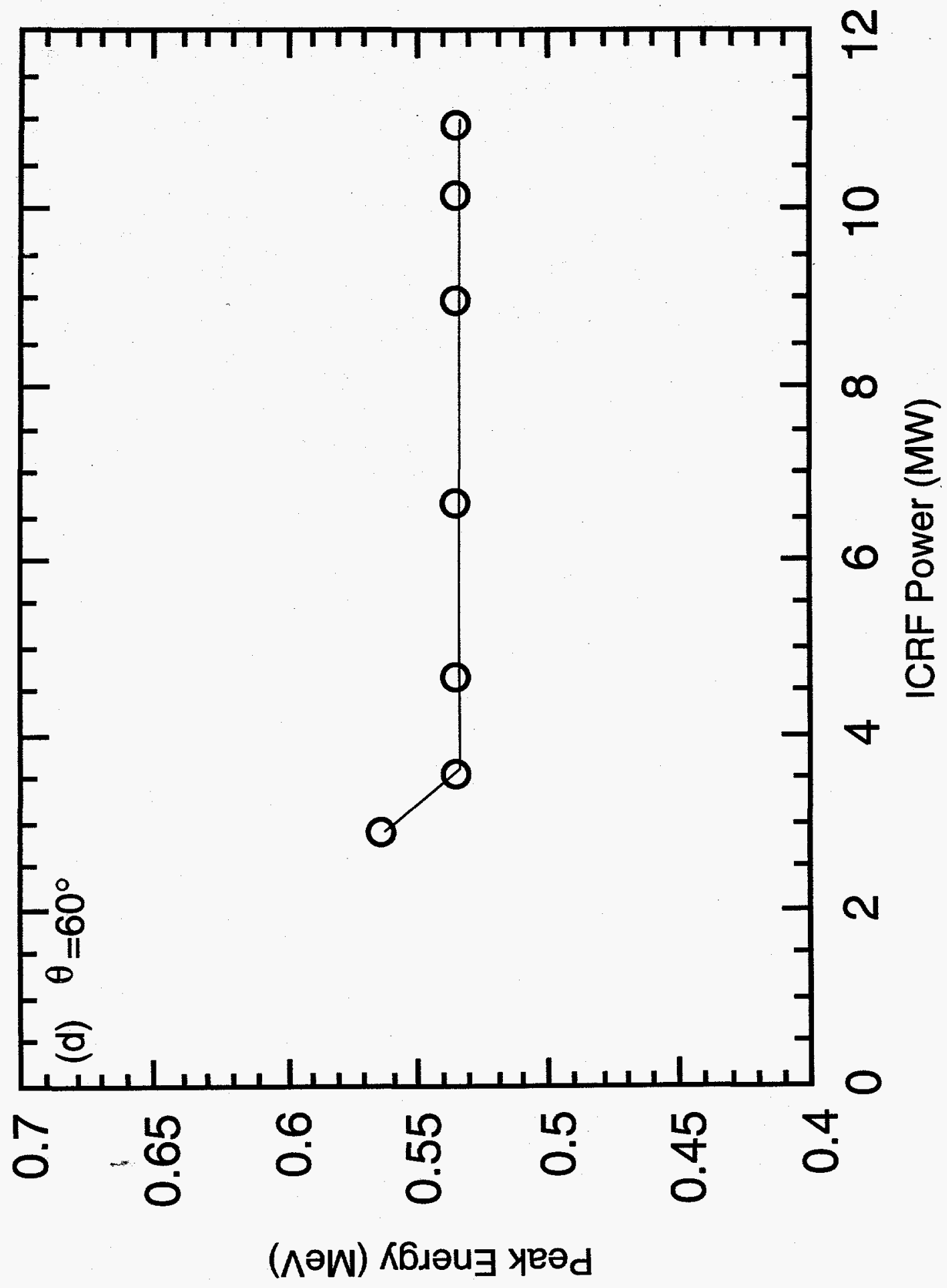

Figure 14 



\section{DISCLAIMER}

This report was prepared as an account of work sponsored by an agency of the United States Government. Neither the United States Government nor any agency thereof, nor any of their employees, makes any warranty, express or implied, or assumes any legal liability or responsibility for the accuracy, completeness, or usefulness of any information, apparatus, product, or process disclosed, or represents that its use would not infringe privately owned rights. Reference herein to any specific commercial product, process, or service by trade name, trademark, manufacturer, or otherwise does not necessarily constitute or imply its endorsement, recommendation, or favoring by the United States Government or any agency thereof. The views and opinions of authors expressed herein do not necessarily state or renlect those of the United States Government or any agency thereof. 
